QUANTUM DYNAMICS

BANACH CENTER PUBLICATIONS, VOLUME 120
INSTITUTE OF MATHEMATICS
POLISH ACADEMY OF SCIENCES
WARSZAWA 2020

\title{
THE ASYMPTOTIC CONNES-MOSCOVICI CHARACTERISTIC MAP AND THE INDEX COCYCLES
}

\author{
ATABEY KAYGUN \\ Istanbul Technical University, Istanbul, Turkey \\ ORCID:0000-0002-9672-6660_E-mail: kaygun@itu.edu.tr \\ SERKAN SÜTLÜ \\ Işik University, Istanbul, Turkey \\ ORCID:0000-0003-0925-8668Ｅ-mail: serkan.sutlu@isikun.edu.tr
}

Dedicated to Paul Baum on his 80th birthday

\begin{abstract}
We show that the (even and odd) index cocycles for theta-summable Fredholm modules are in the image of the Connes-Moscovici characteristic map. To show this, we first define a new range of asymptotic cohomologies, and then we extend the Connes-Moscovici characteristic map to our setting. The ordinary periodic cyclic cohomology and the entire cyclic cohomology appear as two instances of this setup. We then construct an asymptotic characteristic class, defined independently from the underlying Fredholm module. Paired with the $K$-theory, the image of this class under the characteristic map yields a non-zero scalar multiple of the index in the even case, and the spectral flow in the odd case.
\end{abstract}

Introduction. We continue investigating the Connes-Moscovici characteristic map [5, 6] in relation to more geometric invariants of noncommutative spaces. This time, our target is the entire cyclic cohomology of Banach algebras, or more generally of mixed complexes and (co)cyclic objects in the category of Banach spaces [2].

Using Connes' fundamental idea in defining the entire cyclic cohomology [2], we observe a new range of cyclic cohomologies whose cocycles determined by their asymptotic growth. The ordinary periodic and entire cyclic cohomologies are but two specific examples of these asymptotic invariants. Then we observe that the non-trivial asymptotic

2010 Mathematics Subject Classification: Primary 19D55; Secondary 47A53.

Key words and phrases: cyclic cohomology, index theory, Connes-Moscovici characteristic map. The paper is in final form and no version of it will be published elsewhere. 
invariants of noncommutative spaces different from the ordinary periodic cyclic cohomology are beyond the reach of cohomological $\delta$-functors [22, Section 2.1], or more appropriately, cohomological functors defined on the triangulated category of mixed complexes. We refer reader to Proposition 3.3 for the exact statement. This fact has two important consequences. Firstly, asymptotic cohomologies are finer invariants. Secondly, these asymptotic invariants are different from Higson's E-theory [11 or Puschnigg's asymptotic cohomology [19, since both of these invariants come from specific cohomological bifunctors on two different derived categories of Banach spaces using the Connes-Higson asymptotic morphisms [4. Curiously, we also observe that if one were to construct similar asymptotic invariants on Tsygan's cyclic bicomplex, one would obtain drastically different results. We refer the reader to Proposition 3.6 for the details.

Entire cyclic cohomology is crucially useful for the Chern character of the thetasummable Fredholm modules [12, 10, 2]. Our main results in this paper are Theorem 6.7 and Theorem 6.11. where we show that the even and the odd index cocycles of thetasummable Fredholm modules are in the image of an asymptotic analogue of the ConnesMoscovici characteristic map. More explicitly, in Proposition 5.2 we show that there is an asymptotic class, defined independently from the underlying Fredholm module, whose image under a Connes-Moscovici type characteristic homomorphism pairs with $K_{0}$ (resp. $K_{1}$ ) and yields a non-zero scalar multiple of the index (resp. the spectral flow).

The plan of the article. In Section 1 we set the notation and list the basic objects and tools we are going to need in the sequel. In Section 2 we define a range of asymptotic cohomologies, an example of which is the entire cyclic cohomology. In Section 3 we investigate what happens if asymptotic cohomology were to come from a cohomological functor in two cases: first for asymptotic cohomology of mixed complexes, and then for asymptotic cohomology of cyclic bicomplexes. We observe that the results diverge dramatically. The proper context for Connes-Moscovici characteristic map is an appropriate version of the cup product in cohomology. So, we develop such a cup product for asymptotic cohomologies in Section 4. In Section 5 we construct an asymptotic complex out of the geometric $n$-simplices, for $n \geqslant 0$, and show that this complex contains a non-trivial cocycle. Finally, in Section 6 we construct an asymptotic analogue of the Connes-Moscovici characteristic homomorphism explicitly for both the even and the odd theta-summable Fredholm modules. We then apply our machinery to the asymptotic class of Section 5 to obtain the index in the even case, and the spectral flow in the odd case.

Notation and conventions. Throughout the article, we work with complete normed vector spaces over the field of complex numbers $\mathbb{C}$, and the completed tensor product $\widehat{\otimes}$ over $\mathbb{C}$. Since we crucially use norm estimates and their growth, the results of this paper do not immediately generalize to Fréchet spaces, or to more general topological spaces. All (co)cyclic modules are normalized in the sense that the operator norms of the (co)face and (co)degeneracy operators grow at most linearly with respect to their simplicial degree, i.e. they are of asymptotic order $\mathcal{O}(n)$ where $n$ indicates the simplicial degree. The mixed complex of a (co)cyclic module $\mathcal{C}^{\bullet}$ is referred as the $(b, B)$-complex of $\mathcal{C}^{\bullet}$ while Tsygan's bicomplex is simply referred as the cyclic bicomplex of $\mathcal{C}^{\bullet}$. 
Acknowledgments. We would like to thank Kartal Cafe in Beşiktaş, Istanbul for the lively and friendly atmosphere they provided during the planning stages of this article.

\section{Preliminaries}

1.1. The monoidal category of normed (complete) vector spaces. Consider the category of (small) normed vector spaces $\operatorname{Vect}_{\mathbb{C}}^{n}$ over $\mathbb{C}$. Each object $(V,\|\cdot\|)$ consists of a (small) vector space $V$ endowed with a norm $\|\cdot\|$. The underlying metric spaces are not expected to be complete.

There is a full and faithful subcategory $\operatorname{Ban}_{\mathbb{C}}$ of $\operatorname{Vect}_{\mathbb{C}}^{n}$ that consists of complete normed vector spaces.

The usual algebraic tensor product $\otimes$ over $\mathbb{C}$ gives a monoidal product on Vect $_{\mathbb{C}}^{n}$. For a given pair $\left(V,\|\cdot\|_{V}\right)$ and $\left(W,\|\cdot\|_{W}\right)$ of normed vector spaces, we endow $V \otimes W$ with the norm

$$
\|u\|=\inf \left\{\sum_{i}\left\|v_{i}\right\|_{V}\left\|w_{i}\right\|_{W} \mid u=\sum_{i} v_{i} \otimes w_{i}\right\} .
$$

Notice that $\mathbb{C}$, with its standard complex norm, is the unit object in this category.

We note also that since the product of two normed vector spaces has a norm, but is not required to be complete, the subcategory $\mathbf{B a n}_{\mathbb{C}}$ is not a monoidal subcategory. However, one can complete such a product $V \otimes W$ to obtain a complete normed vector space denoted by $V \widehat{\otimes} W$. This construction defines a monoidal product on $\mathbf{B a n}_{\mathbb{C}}$ which is different than that of $\operatorname{Vect}_{\mathbb{C}}^{n}$. For details, see for instance [21, Chapter 2].

In this paper we are going to work with the monoidal category $\left(\mathbf{B a n}_{\mathbb{C}}, \widehat{\otimes}\right)$. We can define algebras, coalgebras, bialgebras and Hopf algebras in the strict monoidal category $\left(\operatorname{Ban}_{\mathbb{C}}, \widehat{\otimes}\right)$. All such objects are assumed to be norm complete and the structure maps (multiplications, comultiplications, units, counits and antipodes) are all bounded (continuous).

1.2. Simplicial and (para)(co)cyclic objects. Let $\Delta, \Delta C$ and $\Delta \mathbb{Z}$ respectively be the simplicial, the cyclic and the paracyclic categories [3, 18. A simplicial object $\mathcal{S}_{\bullet}$ is a functor of the form $\mathcal{S}_{\bullet}: \Delta^{\text {op }} \rightarrow \mathbf{B a n}_{\mathbb{C}}$, and a cyclic object $\mathcal{C}_{\bullet}$ in $\mathbf{B a n}_{\mathbb{C}}$ is a similar functor of the form $\mathcal{C}_{\bullet}: \Delta C^{\text {op }} \rightarrow \mathbf{B a n}_{\mathbb{C}}$. Paracyclic objects are defined similarly to be the functors of the form $\Delta \mathbb{Z}^{\text {op }} \rightarrow \operatorname{Ban}_{\mathbb{C}}$. The "co" versions are obtained by replacing $\Delta^{\mathrm{op}}, \Delta C^{\mathrm{op}}$ and $\Delta \mathbb{Z}^{\mathrm{op}}$ with their categorical duals $\Delta, \Delta C$ and $\Delta \mathbb{Z}$, respectively. As such, again, all structure maps are bounded.

1.3. Mixed complexes. A $\mathbb{N}$-graded vector space $X_{*}$ is called a mixed complex if it is equipped with two different differentials $b_{n}: X_{n} \rightarrow X_{n+1}$ and $B_{n}: X_{n} \rightarrow X_{n-1}$ such that for every $n \in \mathbb{N}$,

$$
b_{n+1} b_{n}=0, \quad B_{n-1} B_{n}=0, \quad \text { and } \quad b_{n-1} B_{n}+B_{n+1} b_{n}=0 .
$$

Given two mixed complexes $\left(X_{*}, b_{*}, B_{*}\right)$ and $\left(X_{*}^{\prime}, b_{*}^{\prime}, B_{*}^{\prime}\right)$, a morphism of mixed complexes $f_{*}: X_{*} \rightarrow X_{*}^{\prime}$ is a graded morphism $f_{*}$ of complexes that yields morphisms of differential graded modules of the form $f_{*}:\left(X_{*}, b_{*}\right) \rightarrow\left(X_{*}^{\prime}, b_{*}^{\prime}\right)$ and $f_{*}:\left(X_{*}, B_{*}\right) \rightarrow\left(X_{*}^{\prime}, B_{*}^{\prime}\right)$. The (small) category of mixed complexes is denoted by $\mathbf{M i x} \mathbf{x}_{\mathbb{C}}$. The category $\mathbf{M i x} \mathbf{x}_{\mathbb{C}}$ is a $\mathbb{C}$-linear abelian exact category [20]. 
1.4. Mixed complex of a cocyclic module. Let $\mathcal{C}^{\bullet}$ be a cocyclic object in Vect $\mathbf{C}_{\mathbb{C}}$, with the cocyclic structure given by

$$
\begin{array}{rlrl}
d_{i}: \mathcal{C}^{n} \longrightarrow \mathcal{C}^{n+1}, & & 0 \leqslant i \leqslant n+1, \\
s_{j}: \mathcal{C}^{n} \longrightarrow \mathcal{C}^{n-1}, & & 0 \leqslant j \leqslant n-1, \\
t_{n}: \mathcal{C}^{n} \longrightarrow \mathcal{C}^{n} . &
\end{array}
$$

Then, $\left(\mathcal{C}^{\bullet}, b_{*}, B_{*}\right)$ with two differentials

$$
b_{n}:=\sum_{i=0}^{n+1}(-1)^{i} d_{i}^{n} \quad \text { and } \quad B_{n}:=N_{n-1} s_{n-1}^{n} t_{n}\left(\operatorname{Id}-(-1)^{n} t_{n}\right) \text {, }
$$

where

$$
N_{n-1}:=\sum_{i=0}^{n-1}(-1)^{i(n-1)} t_{n-1}^{i}
$$

is a mixed complex.

The following proposition is well-known. We refer the reader to [13 for a proof.

Proposition 1.1. Given a cocyclic module $\mathcal{C}^{\bullet}$, let $\mathcal{M}\left(\mathcal{C}^{\bullet}\right):=\left(\mathcal{C}^{*}, b_{*}, B_{*}\right)$. Then the assignment $\mathcal{C}^{\bullet} \mapsto \mathcal{M}\left(\mathcal{C}^{\bullet}\right)$ defines an exact functor from $\Delta C$-Mod, the category of cocyclic modules, to $\mathbf{M i x}_{\mathbb{C}}$, the category of mixed complexes.

1.5. Periodic cyclic cohomology of cocyclic objects. Let $\mathcal{C}^{\bullet}$ be a cocyclic object in Vect $_{\mathbb{C}}$, whose cocyclic structure is given as in (1.1). The periodic cyclic cohomology of $\mathcal{C}^{\bullet}$ is the direct sum total homology of the cyclic bicomplex, i.e. the upper half plane bicomplex $\mathcal{C C}^{p, q}:=\mathcal{C}^{q}$ with $p, q \in \mathbb{Z}, q \geqslant 0$,

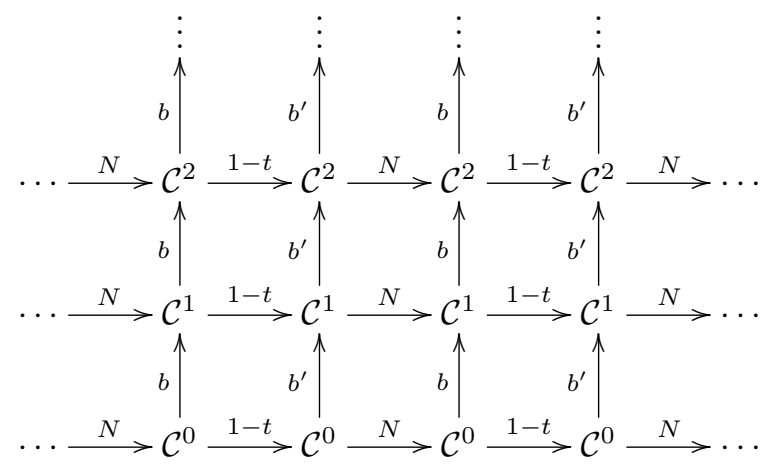

where

$$
b:=\sum_{i=0}^{n+1}(-1)^{i} d_{i}^{n}, \quad b^{\prime}:=\sum_{i=0}^{n}(-1)^{i} d_{i}^{n}, \quad N_{n}:=\sum_{i=0}^{n}(-1)^{n i} t_{n}^{i},
$$

In other words,

$$
H P^{*}\left(\mathcal{C}^{\bullet}\right):=H_{*}\left(\operatorname{Tot}_{\oplus} \mathcal{C C} \bullet \bullet\right)
$$

is given by the periodic differential graded complex

$$
\operatorname{Tot}_{\oplus}^{i} \mathcal{C C} \mathcal{C}^{\bullet, \bullet}:=\bigoplus_{n \geqslant 0} \mathcal{C}^{n} \quad \text { with } \quad \partial_{i}: \operatorname{Tot}_{\oplus}^{i} \mathcal{C} \mathcal{C}^{\bullet, \bullet} \rightarrow \operatorname{Tot}_{\oplus}^{1-i} \mathcal{C C} \mathcal{C}^{\bullet, \bullet}
$$

for $i \in\{0,1\}$, which are called the even and the odd cochain groups respectively. 
Allowing the direct products in (1.3) rather than the direct sums, one defines the spaces

$$
\operatorname{Tot}_{\infty}^{i} \mathcal{C C}^{\bullet, \bullet}:=\prod_{n \geqslant 0} \mathcal{C}^{n}, \quad i \in\{0,1\},
$$

of even and odd cochains with infinite support, and hence the direct product total

$$
\ldots \longrightarrow \operatorname{Tot}_{\infty}^{0} \mathcal{C C} \mathcal{C}^{\bullet, \bullet} \stackrel{\partial_{0}}{\longrightarrow} \operatorname{Tot}_{\infty}^{1} \mathcal{C C} \mathcal{C}^{\bullet, \bullet} \stackrel{\partial_{1}}{\longrightarrow} \operatorname{Tot}_{\infty}^{0} \mathcal{C C} \mathcal{C}^{\bullet, \bullet} \stackrel{\partial_{0}}{\longrightarrow} \operatorname{Tot}_{\infty}^{1} \mathcal{C C}^{\bullet, \bullet} \stackrel{\partial_{1}}{\longrightarrow} \ldots
$$

The homology of the latter is called the periodic cyclic cohomology with infinite support

$$
H P_{\infty}^{*}\left(\mathcal{C}^{\bullet}\right):=H_{*}\left(\operatorname{Tot}_{\infty} \mathcal{C} \mathcal{C}^{\bullet \bullet}\right)
$$

We note from [2, 14] that this homology is trivial for every cocyclic module $\mathcal{C}^{\bullet}$.

1.6. Periodic cyclic cohomology of mixed complexes. Let $\left(\mathcal{C}^{*}, b_{*}, B_{*}\right)$ be a mixed complex. We can construct a bicomplex $\mathcal{C C}^{p, q}:=\mathcal{C}^{q+p}$ with $p, q \in \mathbb{Z}$ such that $q \geqslant-p$, given by

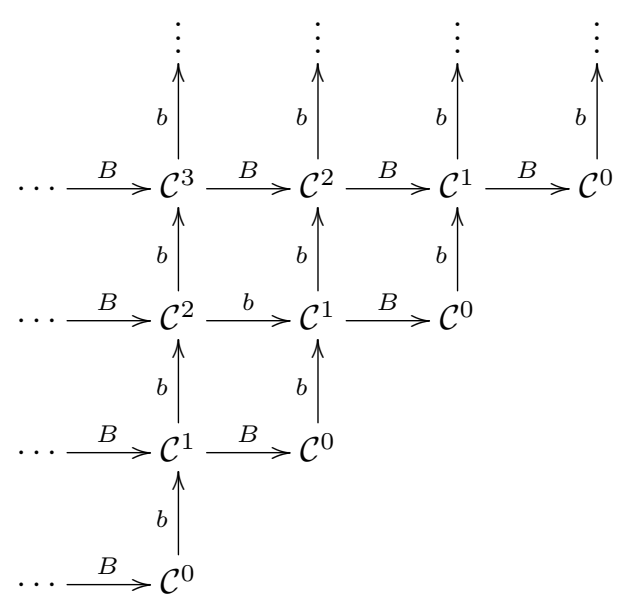

Similar to the bicomplexes defined in Subsection 1.5 we again have

$$
\operatorname{Tot}_{\oplus}^{i} \mathcal{C} \mathcal{C}^{\bullet \bullet \bullet}:=\bigoplus_{n \geqslant 0} \mathcal{C}^{n} \quad \text { and } \quad \operatorname{Tot}_{\infty}^{i} \mathcal{C} \mathcal{C}^{\bullet \bullet \bullet}:=\prod_{n \geqslant 0} \mathcal{C}^{n}, \quad i \in\{0,1\}
$$

together with the differentials $\partial^{i}=b+B$ with $i=0,1$. These complexes give us $H P^{*}\left(\mathcal{C}^{*}\right)$ and $H P_{\infty}^{*}\left(\mathcal{C}^{*}\right)$, respectively.

In case $\mathcal{C}^{\bullet}$ is a cocyclic object such that the corresponding bar complex $\left(\mathcal{C}^{*}, b^{\prime}\right)$ is acyclic, then the double complex given in $(1.2)$ and the double complex given in $(1.5)$ are quasi-isomorphic in both direct sum and direct product versions. See [14 for details.

2. Asymptotic hierarchy and cohomology. As is observed in [2, 12, 14, 16, the cohomology of the subcomplex of cochains of infinite support satisfying certain growth conditions might be nontrivial. In the following section, we are going to consider the subcomplexes of cochains with specific growth conditions. 
2.1. Asymptotic hierarchy of sequences. Given two sequences $\left(x_{n}\right)_{n \geqslant 0}$ and $\left(y_{n}\right)_{n \geqslant 0}$ of real numbers in $(0, \infty)$, we define

$$
\mathcal{E}\left(y_{n}\right):=\left\{\left(x_{n}\right)_{n \geqslant 0} \mid\left(x_{n}\right)_{n \geqslant 0} \prec\left(y_{n}\right)_{n \geqslant 0}\right\},
$$

where we write $\left(x_{n}\right)_{n \geqslant 0} \prec\left(y_{n}\right)_{n \geqslant 0}$ if

$$
\limsup _{n \rightarrow \infty} \frac{r^{n} x_{n}}{y_{n}}=0
$$

for every $r \in(0, \infty)$.

We leave the proof of the following simple lemmas to the reader.

LEMmA 2.1. Let $\left(x_{n}\right)_{n \geqslant 0}$ and $\left(y_{n}\right)_{n \geqslant 0}$ be two sequences in $(0, \infty)$. Then $\left(x_{n}\right)_{n \geqslant 0} \prec$ $\left(y_{n}\right)_{n \geqslant 0}$ if and only if

$$
\limsup _{n \rightarrow \infty} \sqrt[n]{\frac{x_{n}}{y_{n}}}=0 .
$$

LEMMA 2.2. Let $\left(x_{n}\right)_{n \geqslant 0}$ and $\left(y_{n}\right)_{n \geqslant 0}$ be two sequences in $(0, \infty)$. Then the radius of convergence of the power series $\sum_{n \geqslant 0} \frac{x_{n}}{y_{n}} z^{n}$ is infinite if and only if $\left(x_{n}\right) \prec\left(y_{n}\right)$.

REMARK 2.3. The asymptotic decay condition (2.1) is quite strong. For example, $\mathcal{E}\left(\lambda^{n}\right)=$ $\mathcal{E}(1)$ and $\mathcal{E}\left(n^{a}\right)=\mathcal{E}(1)$ for every $a, \lambda \in[0, \infty)$.

2.2. Asymptotic cohomology of cocyclic objects. Assume $\mathcal{C}^{\bullet}$ is a cocyclic object in $\mathbf{B a n}_{\mathbb{C}}$. We define a graded subspace of $C_{\infty}^{*}\left(\mathcal{C}^{\bullet}\right)$ by letting

$$
C_{\mathcal{E}\left(x_{n}\right)}^{i}\left(\mathcal{C}^{\bullet}\right):=\left\{\left(\varphi_{2 n+i}\right)_{n \geqslant 0} \in C_{\infty}^{i}\left(\mathcal{C}^{\bullet}\right) \mid\left(\left\|\varphi_{2 n+i}\right\|\right)_{n \geqslant 0} \in \mathcal{E}\left(x_{n}\right)\right\}, \quad i=0,1 .
$$

On the other extreme, setting $C_{\oplus}^{i}\left(\mathcal{C}^{\bullet}\right):=\operatorname{Tot}_{\oplus}^{i} \mathcal{C} \mathcal{C}^{\bullet \bullet} \subseteq \operatorname{Tot}_{\infty}^{i} \mathcal{C C}^{\bullet} \bullet$ the direct sum total computing the usual (algebraic) periodic cyclic cohomology of $\mathcal{C}^{\bullet}$, we get $C_{\oplus}^{i}\left(\mathcal{C}^{\bullet}\right) \subseteq$ $C_{\mathcal{E}\left(x_{n}\right)}^{i}\left(\mathcal{C}^{\bullet}\right)$ for any $\left(x_{n}\right)_{n \geqslant 0}$, and $i=0,1$.

Now, given a sequence $\left(x_{n}\right)_{n \geqslant 0}$, we wish $C_{\mathcal{E}\left(x_{n}\right)}^{i}\left(\mathcal{C}^{\bullet}\right)$ to be a subcomplex of $C_{\infty}^{*}\left(\mathcal{C}^{\bullet}\right)$. Then we need to show that the graded space $(2.3)$ is closed under the differentials $b$ and $B$ which, in turn, requires an estimate for the operator norm of these operators.

We first note that since the cyclic operators $t_{n}$ satisfy $t_{n}^{n+1}=\mathrm{Id}$, it follows at once that $\left\|t_{n}\right\|^{n}=1$, and hence $\left\|t_{n}\right\|=1$. Also, since the norm operator is defined as $N_{n}=$ $\sum_{i=0}^{n}(-1)^{n i} t_{n}^{i}$, we have $\left\|N_{n}\right\| \leqslant n+1$. On the other hand, if $\mathcal{C}^{\bullet}$ were the standard cocyclic object associated to a unital Banach algebra, see for instance [2, 14], the coface operators $d_{i}: \mathcal{C}^{n} \rightarrow \mathcal{C}^{n+1}$ would be given by

$$
d_{i} \varphi_{n}\left(a_{0}, \ldots, a_{n+1}\right)=\varphi_{n}\left(a_{0}, \ldots, a_{i} a_{i+1}, \ldots, a_{n+1}\right)
$$

and hence we would have $\left\|d_{i} \varphi_{n}\right\|=\left\|\varphi_{n}\right\|$, for any $\varphi_{n} \in \mathcal{C}^{n}$. Since $b_{n}:=\sum_{i=0}^{n+1}(-1)^{i} d_{i}$ and $b_{n}^{\prime}:=\sum_{i=0}^{n}(-1)^{i} d_{i}$, we would have obtained the estimates

$$
\left\|b_{n} \varphi_{n}\right\| \leqslant(n+2)\left\|\varphi_{n}\right\| \quad \text { and } \quad\left\|b_{n}^{\prime} \varphi_{n}\right\| \leqslant(n+1)\left\|\varphi_{n}\right\|
$$

for any $\varphi_{n} \in \mathcal{C}^{n}$ [14, Lemma 2.1.2]. However, we do not have these bounds on the norms of the coface operators for an arbitrary cocyclic module $\mathcal{C} \bullet$. Nevertheless, the coface maps $d_{i}^{n}$, the codegeneracy maps $s_{j}^{n}$ and the cyclic maps $t_{n}$ satisfy the relations

$$
d_{i}^{n}=t_{n+1}^{i} d_{0}^{n} t_{n}^{-i}, \quad \text { and } \quad s_{j}^{n}=t_{n}^{-i} s_{0}^{n} t_{n+1}^{i}
$$


since $\mathcal{C}^{\bullet}$ is a cocyclic object [18, 3]. As a result, we can control the norms of the coface and codegeneracy operators by controlling only the norm of the 0 -th coface and 0 -th codegeneracy operators on each grade. Accordingly, we give the following definition.

Definition 2.4. A cocyclic object $\mathcal{C}^{\bullet}$ with coface maps $d_{i}^{n}$ and codegeneracy maps $s_{j}^{n}$ for $0 \leqslant i \leqslant n$ and $0 \leqslant j \leqslant n-1$, is called asymptotically normalized if $\left\|d_{0}^{n}\right\|=1=\left\|s_{0}^{n}\right\|$ for any $n \geqslant 0$.

Let $\left(\mathcal{C}^{\bullet}, d_{i}^{n}, s_{j}^{n}, t_{n}\right)$ be a cocyclic object. Then $\left(\mathcal{C}^{\bullet}, \frac{d_{i}^{n}}{\left\|d_{0}^{n}\right\|}, \frac{s_{j}^{n}}{\left\|s_{0}^{n}\right\|}, t_{n}\right)$ satisfies all cocyclic identities except

$$
\sigma_{j}^{n+1} \delta_{i}^{n}=\mathrm{Id}, \quad \text { for } i=j \text { or } i=j+1 \text {. }
$$

Instead, setting $\delta_{i}^{n}:=\frac{d_{i}^{n}}{\left\|d_{0}^{n}\right\|}$ and $\sigma_{j}^{n}:=\frac{s_{j}^{n}}{\left\|s_{0}^{n}\right\|}$, we get

$$
\sigma_{j}^{n+1} \delta_{i}^{n}=\frac{1}{\left\|d_{0}^{n}\right\|\left\|s_{0}^{n}\right\|} \mathrm{Id}, \quad \text { for } i=j \text { or } i=j+1 .
$$

However, the automorphism $\psi_{n}: \mathcal{C}^{n} \longrightarrow \mathcal{C}^{n}$ defined as $\psi_{n}:=\left\|d_{0}^{n}\right\|\left\|s_{0}^{n}\right\|$ Id commutes with all structure maps:

$$
\psi_{n+1} \circ \delta_{i}^{n}=\delta_{i}^{n} \circ \psi_{n}, \quad \psi_{n-1} \circ \sigma_{j}^{n}=\sigma_{j}^{n} \circ \psi_{n}, \quad \psi_{n-1} \circ t_{n}=t_{n} \circ \psi_{n} .
$$

Hence, the associated mixed complex

$$
\left(\mathcal{C}^{\bullet}, b:=\sum_{i=0}^{n+1}(-1)^{i} d_{i}^{n}, B:=N_{n-1} s_{n-1}^{n} t_{n}\left(\operatorname{Id}-(-1)^{n} t_{n}\right)\right)
$$

is isomorphic to the mixed complex

$$
\left(\mathcal{C}^{\bullet}, \widetilde{b}:=\sum_{i=0}^{n+1}(-1)^{i} \delta_{i}^{n}, \widetilde{B}:=N_{n-1} \sigma_{n-1}^{n} t_{n}\left(\operatorname{Id}-(-1)^{n} t_{n}\right)\right) .
$$

As a result, from this point on we assume that all (co)cyclic modules are asymptotically normalized.

Proposition 2.5. Given a cocyclic object $\mathcal{C}^{\bullet}$ in $\mathbf{B a n}_{\mathbb{C}}$, and a sequence $\left(x_{n}\right)_{n \geqslant 0}$ in $(0, \infty)$, the graded space $C_{\mathcal{E}\left(x_{n}\right)}^{*}\left(\mathcal{C}^{\bullet}\right)$ is a differential graded subspace of the periodic cyclic complex with infinite support $C_{\infty}^{*}\left(\mathcal{C}^{\bullet}\right)$.

Proof. Let $\varphi=\left(\varphi_{2 n+i}\right)_{n \geqslant 0} \in C_{\mathcal{E}\left(x_{n}\right)}^{i}$ i.e. $\left(\left\|\varphi_{2 n+i}\right\|\right)_{n \geqslant 0} \in \mathcal{E}\left(x_{n}\right)$. Then for any $r \in(0, \infty)$,

$$
\begin{aligned}
\limsup _{n \rightarrow \infty} \frac{\left\|b_{2 n+i} \varphi_{2 n+i}\right\| r^{n}}{x_{n}} & \leqslant \limsup _{n \rightarrow \infty} \frac{\left\|b_{2 n+i}\right\|\left\|\varphi_{2 n+i}\right\| r^{n}}{x_{n}} \leqslant \limsup _{n \rightarrow \infty} \frac{(2 n+1+i)\left\|\varphi_{2 n+i}\right\| r^{n}}{x_{n}} \\
& \leqslant \limsup _{n \rightarrow \infty} \frac{2^{n}\left\|\varphi_{2 n+i}\right\| r^{n}}{x_{n}}=\limsup _{n \rightarrow \infty} \frac{\left\|\varphi_{2 n+i}\right\|(2 r)^{n}}{x_{n}}=0 .
\end{aligned}
$$

In other words, $b \varphi \in C_{\mathcal{E}\left(x_{n}\right)}^{1-i}$. The proof works for the operator $B$ mutatis mutandis.

We are going to use $H P_{\mathcal{E}\left(x_{n}\right)}^{*}\left(\mathcal{C}^{\bullet}\right)$ to denote the cohomology of this subcomplex.

2.3. Asymptotic cohomology of mixed complexes. There is an analogous asymptotic cohomology for mixed complexes. For a more detailed treatment on the subject, for the specific case of the entire cohomology, we refer the reader to [16]. 
Given a mixed complex $\left(X_{*}, b_{*}, B_{*}\right)$ one has the differential complexes

$$
C_{\infty}^{i}\left(X_{*}\right)=\prod_{n=0}^{\infty} X_{n} \quad \text { and } \quad C^{i}\left(X_{*}\right)=\bigoplus_{n=0}^{\infty} X_{n}
$$

together with the differential $b+B$. Similar to the asymptotic differential complex $C_{\mathcal{E}\left(x_{n}\right)}^{*}\left(\mathcal{C}^{\bullet}\right)$ associated to a cocyclic module $\mathcal{C}^{\bullet}$, for any sequence of non-negative real numbers $\left(x_{n}\right)_{n \geqslant 0}$ one can also define the differential subcomplex

$$
C_{\mathcal{E}\left(x_{n}\right)}^{i}\left(X_{*}\right):=\left\{\left(\varphi_{2 n+i}\right)_{n \geqslant 0} \in C_{\infty}^{i}\left(X_{*}\right) \mid\left\|\varphi_{2 n+i}\right\| \prec\left(x_{n}\right)\right\} .
$$

2.4. Entire cyclic cohomology as asymptotic cohomology. As is indicated in Proposition 2.5 now there is a whole gamut of asymptotic subcomplexes between $C_{\oplus}^{*}\left(\mathcal{C}^{\bullet}\right)$ and $C_{\infty}^{*}\left(\mathcal{C}^{\bullet}\right)$ for a given cocyclic object $\mathcal{C}^{\bullet}$.

We recall from [14], see also [2, that given a cocyclic object $\mathcal{C}^{\bullet}$ in $\mathbf{B a n}_{\mathbb{C}}$, an even (resp. odd) infinite cochain $\left(\varphi_{2 n}\right)_{n \geqslant 0} \in C_{\infty}^{0}\left(\mathcal{C}^{\bullet}\right)\left(\right.$ resp. $\left.\left(\varphi_{2 n+1}\right)_{n \geqslant 0} \in C_{\infty}^{1}\left(\mathcal{C}^{\bullet}\right)\right)$ is called entire if the radius of convergence of the power series

$$
\sum_{n \geqslant 0} \frac{(2 n) !\left\|\varphi_{2 n}\right\|}{n !} z^{n} \quad\left(\text { resp. } \sum_{n \geqslant 0} \frac{(2 n+1) !\left\|\varphi_{2 n+1}\right\|}{n !} z^{n}\right)
$$

is infinite. Following the common notation of $C_{\varepsilon}^{i}\left(\mathcal{C}^{\bullet}\right), i=0,1$, for the space of (even, and odd) entire cochains, we have

$$
C_{\varepsilon}^{i}\left(\mathcal{C}^{\bullet}\right)=C_{\mathcal{E}(n ! /(2 n) !)}^{i}\left(\mathcal{C}^{\bullet}\right) \subset C_{\mathcal{E}(1)}^{i}\left(\mathcal{C}^{\bullet}\right)
$$

In particular, if $\mathcal{C}^{\bullet}$ is the standard cocyclic object associated to a unital Banach algebra $A$, then $C_{\varepsilon}^{*}\left(\mathcal{C}^{\bullet}\right)$ is precisely the Connes' entire subcomplex $C_{\varepsilon}^{*}(A)$, and $H P_{\varepsilon}^{*}\left(\mathcal{C}^{\bullet}\right)$ is nothing but the entire cyclic cohomology $H P_{\varepsilon}(A)$ of the algebra $A$.

In case $\mathcal{C}^{\bullet}$ is the cocyclic object associated to a (Banach-)Hopf algebra $H$, together with a modular pair $(\delta, \sigma)$ in involution, [5], then we shall call $C_{\varepsilon}^{*}\left(\mathcal{C}^{\bullet}\right)$ the entire Hopfcyclic complex of $H$, and $H P_{\varepsilon}^{*}\left(\mathcal{C}^{\bullet}\right)$ the entire Hopf-cyclic cohomology of $H$. More generally, we will use the notation $C_{\mathcal{E}\left(x_{n}\right)}^{*}(H, \delta, \sigma)$ to denote the asymptotic Hopf-cyclic complex associated with the sequence $\left(x_{n}\right)_{n \geqslant 0}$, and $\operatorname{HP}_{\mathcal{E}\left(x_{n}\right)}^{*}(H, \delta, \sigma)$ for the cohomology of this complex.

\section{Cohomological functors and asymptotic cohomology}

3.1. Cohomological functors on the category of mixed complexes. A $\mathbb{Z}$-graded functor of the form $F_{*}: \mathbf{M i x}_{\mathbb{C}} \rightarrow$ Vect $_{\mathbb{C}}$ is called a cohomological $\delta$-functor $[22$ if every short exact sequence of mixed complexes

$$
0 \rightarrow\left(X_{*}, b_{*}, B_{*}\right) \rightarrow\left(X_{*}^{\prime}, b_{*}^{\prime}, B_{*}^{\prime}\right) \rightarrow\left(X_{*}^{\prime \prime}, b_{*}^{\prime \prime}, B_{*}^{\prime \prime}\right) \rightarrow 0
$$

is sent to a long exact sequence of $\mathbb{C}$-modules

$$
\cdots \rightarrow F_{n}\left(X_{*}, b_{*}, B_{*}\right) \rightarrow F_{n}\left(X_{*}^{\prime}, b_{*}^{\prime}, B_{*}^{\prime}\right) \rightarrow F_{n}\left(X_{*}^{\prime \prime}, b_{*}^{\prime \prime}, B_{*}^{\prime \prime}\right) \rightarrow F_{n+1}\left(X_{*}, b_{*}, B_{*}\right) \rightarrow \cdots
$$

Given a cohomological $\delta$-functor $F_{*}: \operatorname{Mix}_{\mathbb{C}} \rightarrow \operatorname{Vect}_{\mathbb{C}}$, a mixed complex $\left(X_{*}, b_{*}, B_{*}\right)$ is called $F$-acyclic if $F_{n}\left(X_{*}, b_{*}, B_{*}\right)=0$ for every $n \in \mathbb{Z}$. Similarly, we are going to call a morphism of mixed complexes $f_{*}:\left(X_{*}, b_{*}, B_{*}\right) \rightarrow\left(X_{*}^{\prime}, b_{*}^{\prime}, B_{*}^{\prime}\right)$ a $F$-equivalence if 
the induced morphisms of $\mathbb{C}$-modules $F_{n}\left(f_{*}\right): F_{n}\left(X_{*}, b_{*}, B_{*}\right) \rightarrow F_{n}\left(X_{*}^{\prime}, b_{*}^{\prime}, B_{*}^{\prime}\right)$ are all isomorphisms.

Proposition 3.1. Let $\left(X_{*}, b_{*}, B_{*}\right)$ be a mixed complex such that $\left(X_{*}, b_{*}\right)$ is acyclic. Then $\left(X_{*}, b_{*}, B_{*}\right)$ is F-acyclic for any cohomological $\delta$-functor $F_{*}: \mathbf{M i x}_{\mathbb{C}} \rightarrow \mathbf{V e c t}_{\mathbb{C}}$.

Proof. There is a short exact sequence of mixed complexes of the form

$$
0 \rightarrow\left(\operatorname{ker}\left(b_{*}\right), 0, B_{*}\right) \rightarrow\left(X_{*}, b_{*}, B_{*}\right) \stackrel{ \pm b_{*}}{\longrightarrow}\left(i m\left(b_{*}\right), 0, B_{*}\right) \rightarrow 0 .
$$

Then for a cohomological $\delta$-functor $F_{*}: \mathbf{M i x}_{\mathbb{C}} \rightarrow \operatorname{Vect}_{\mathbb{C}}$ we get a long exact sequence of the form

$$
\begin{aligned}
\cdots \rightarrow F_{n-1}\left(i m\left(b_{*}\right), 0, B_{*}\right) \rightarrow & F_{n}\left(\operatorname{ker}\left(b_{*}\right), 0, B_{*}\right) \rightarrow F_{n}\left(X_{*}, b_{*}, B_{*}\right) \rightarrow \\
& \rightarrow F_{n}\left(i m\left(b_{*}\right), 0, B_{*}\right) \rightarrow F_{n+1}\left(\operatorname{ker}\left(b_{*}\right), 0, B_{*}\right) \rightarrow \cdots .
\end{aligned}
$$

By acyclicity of $\left(X_{*}, b_{*}\right)$, the natural embedding $i m\left(b_{*}\right) \rightarrow \operatorname{ker}\left(b_{*}\right)[+1]$ is bijective. Thus we get $F_{n}\left(X_{*}, b_{*}, B_{*}\right)=0$ for every $n \in \mathbb{Z}$.

Proposition 3.2. Let $f_{*}:\left(X_{*}, b_{*}, B_{*}\right) \rightarrow\left(X_{*}^{\prime}, b_{*}^{\prime}, B_{*}^{\prime}\right)$ be a morphism of mixed complexes such that $f_{*}:\left(X_{*}, b_{*}\right) \rightarrow\left(X_{*}^{\prime}, b_{*}^{\prime}\right)$ is a quasi-isomorphism. Then $f_{*}$ is an F-equivalence for every cohomological $\delta$-functor $F: \mathbf{M i x}_{\mathbb{C}} \rightarrow \operatorname{Vect}_{\mathbb{C}}$.

Proof. The abelian $\mathbb{C}$-linear category of mixed complexes $\mathbf{M i x} \mathbb{C}$ is isomorphic to the abelian $\mathbb{C}$-linear category of differential graded modules over the quotient polynomial $\mathbb{C}$-algebra $\mathcal{B}:=\mathbb{C}[B] /\left(B^{2}\right)$. Given a morphism $f_{*}: X_{*} \rightarrow X_{*}^{\prime}$ of differential graded $\mathcal{B}$-modules, the fact that $f_{*}$ is a quasi-isomorphism in the $b_{*}$-direction is equivalent to the fact that $f_{*}$ is an ordinary quasi-isomorphism of differential graded $\mathcal{B}$-modules. Now, we form the mapping cone $C\left(f_{*}\right)$ and consider

$$
0 \rightarrow\left(X_{*}, b_{*}\right) \rightarrow C\left(f_{*}\right) \rightarrow K\left(f_{*}\right) \rightarrow 0
$$

where $K\left(f_{*}\right)$ is the cokernel of the embedding $\left(X_{*}, b_{*}\right) \rightarrow C\left(f_{*}\right)$. Since $f_{*}$ is a quasiisomorphism, $K\left(f_{*}\right)$ is acyclic. Then the result follows from Proposition 3.1

3.2. Bounded mixed complexes. For a given index $n \in \mathbb{Z}$ and a mixed complex $\left(X_{*}, b_{*}, B_{*}\right)$, the good truncations $\left(X_{* \leqslant n}, b_{*}, B_{*}\right)$ and $\left(X_{*>n}, b_{*}, B_{*}\right)$ of $X_{*}$ are defined to be

$$
X_{m \leqslant n}=\left\{\begin{array}{ll}
X_{m} & \text { if } m \leqslant n \\
i m\left(b_{m}\right) & \text { if } m=n+1 \\
0 & \text { otherwise }
\end{array} \quad \text { and } \quad X_{m>n}= \begin{cases}0 & \text { if } m \leqslant n \\
X_{m+1} / i m\left(b_{m}\right) & \text { if } m=n+1 \\
X_{m} & \text { otherwise }\end{cases}\right.
$$

These mixed complexes fit into a short exact sequence of the form

$$
0 \rightarrow\left(X_{* \leqslant n}, b_{*}, B_{*}\right) \rightarrow\left(X_{*}, b_{*}, B_{*}\right) \rightarrow\left(X_{*>n}, b_{*}, B_{*}\right) \rightarrow 0 .
$$

For the homology in the $b_{*}$ direction, we get

$$
H_{m}\left(X_{* \leqslant n}, b_{*}\right)= \begin{cases}H_{m}\left(X_{*}, b_{*}\right) & \text { if } m \leqslant n \\ 0 & \text { otherwise }\end{cases}
$$


and

$$
H_{m}\left(X_{*>n}, b_{*}\right)= \begin{cases}H_{m}\left(X_{*}, b_{*}\right) & \text { if } m>n \\ 0 & \text { otherwise. }\end{cases}
$$

A mixed complex $\left(X_{*}, b_{*}, B_{*}\right)$ is called bounded if there is an index $N$ such that the good truncation $\left(X_{*>N}, b_{*}\right)$ is acyclic. Similarly, a (co)cyclic object $\mathcal{C}^{\bullet}$ is called bounded if its associated mixed complex $\mathcal{M}\left(\mathcal{C}^{\bullet}\right)$ is bounded.

Proposition 3.3. If $H P_{\mathcal{E}\left(x_{n}\right)}^{*}$ is a cohomological $\delta$-functor on the category of mixed complexes, then $H P_{\mathcal{E}\left(x_{n}\right)}^{*}$ must be the same as the algebraic periodic cohomology $H P^{*}$ on the subcategory of bounded mixed complexes.

Proof. Assume we have a bounded mixed complex $\left(X_{*}, b_{*}, B_{*}\right)$. Then for $n$ large enough, the natural embedding of mixed complexes $\left(X_{* \leqslant n}, b_{*}, B_{*}\right) \rightarrow\left(X_{*}, b_{*}, B_{*}\right)$ induces a homotopy equivalence of mixed complexes since $\left(X_{* \leqslant n}, b_{*}\right) \rightarrow\left(X_{*}, b_{*}\right)$ is a quasi-isomorphism. On the other hand, in view of the assumption, replacing $\left(X_{*}, b_{*}, B_{*}\right)$ with its good truncation $\left(X_{* \leqslant n}, b_{*}, B_{*}\right)$ for a large enough $n$, we obtain a homotopical equivalence of the form

$$
C_{\mathcal{E}\left(x_{n}\right)}^{*}\left(X_{* \leqslant n}, b_{*}, B_{*}\right) \stackrel{\simeq}{\longrightarrow} C_{\mathcal{E}\left(x_{n}\right)}^{*}\left(X_{*}, b_{*}, B_{*}\right) .
$$

However, the bicomplex computing $C_{\mathcal{E}\left(x_{n}\right)}^{*}\left(X_{* \leqslant n}, b_{*}, B_{*}\right)$ is confined within a bounded strip along the $p=q$ line. This means $C_{\mathcal{E}\left(x_{n}\right)}^{*}\left(X_{* \leqslant n}, b_{*}, B_{*}\right)$ is equal to the algebraic periodic complex $C^{*}\left(X_{* \leqslant n}, b_{*}, B_{*}\right)$ for large enough $n$. On the other hand, $C^{*}\left(X_{* \leqslant n}, b_{*}, B_{*}\right)$ is homotopy equivalent to $C^{*}\left(X_{*}, b_{*}, B_{*}\right)$ since the algebraic periodic cyclic cohomology $H P^{*}$ is a cohomological $\delta$-functor on bounded mixed complexes. This follows from the fact that the ordinary cyclic cohomology $H C^{*}$ of cocyclic modules is a cohomological $\delta$-functor [13, Proposition 1.3], and that the periodic cyclic cohomology is the stabilization of $H C^{*}$ under the periodicity operator $S$. Then for large enough $m, H C^{m}\left(X_{*}\right)$ and $H P^{m(\bmod 2)}\left(X_{*}\right)$ are the same for bounded mixed complexes. The result follows.

3.3. The cyclic bicomplex and the stability phenomenon. Instead of using the $(b, B)$-complex of Connes, one can use the cyclic bicomplex in studying cyclic cohomology. In this subsection, we will follow this route.

Let $\mathcal{C}^{\bullet}$ be a asymptotically normalized cocyclic object in $\mathbf{B a n}_{\mathbb{C}}$, and consider the cyclic bicomplex

$$
C^{p, q}=C^{q}, \quad d_{v}^{p, q}=\left\{\begin{array}{ll}
b_{q} & \text { if } p \text { is even, } \\
b_{q}^{\prime} & \text { if } p \text { is odd },
\end{array} \quad d_{h}^{p, q}= \begin{cases}\left(1-t_{q}\right) & \text { if } p \text { is even } \\
N_{q} & \text { if } p \text { is odd }\end{cases}\right.
$$

One can similarly define the product total complex

$$
\operatorname{Tot}_{\Pi}^{n}\left(\mathcal{C}^{\bullet}\right)=\prod_{m=0}^{\infty} C^{m}
$$

together with the differential $d_{n}$ : $\operatorname{Tot}_{\Pi}^{n} \rightarrow \operatorname{Tot}_{\Pi}^{n+1}$ coming from $d_{v}^{*, *}$ and $d_{h}^{*, *}$. We now define asymptotic subcomplexes by imposing a growth condition:

$$
\operatorname{Tot}_{\mathcal{E}\left(x_{n}\right)}^{n}\left(\mathcal{C}^{\bullet}\right)=\left\{\left(\varphi_{n}\right)_{n \geqslant 0} \in \prod_{m=0}^{\infty} C^{m} \mid\left(\left\|\varphi_{n}\right\|\right)_{n \geqslant 0} \in \mathcal{E}\left(x_{n}\right)\right\} .
$$


The cohomology of this new complex yields a different asymptotic cohomology theory for cocyclic objects. We shall reserve the notation $H S_{\mathcal{E}\left(x_{n}\right)}^{*}$ to this new cohomology theory.

Let us consider the following definition.

Definition 3.4. A morphism of cocyclic modules $f^{\bullet}: \mathcal{C}^{\bullet} \rightarrow \mathcal{D}^{\bullet}$ is called a stable isomorphism if there is an index $N \geqslant 0$ such that the induced morphism $H^{n}\left(f^{\bullet}\right): H^{n}\left(\mathcal{C}^{\bullet}\right) \rightarrow$ $H^{n}\left(\mathcal{D}^{\bullet}\right)$ on the Hochschild cohomology is an isomorphism for every $n \geqslant N$.

Proposition 3.5. Let $f^{\bullet}: \mathcal{C}^{\bullet} \rightarrow \mathcal{D}^{\bullet}$ be a stable isomorphism of cocyclic objects. If $H S_{\mathcal{E}\left(x_{n}\right)}^{*}$ is a cohomological functor, then $f^{\bullet}$ induces an isomorphism in cohomology

$$
H S_{\mathcal{E}\left(x_{n}\right)}^{*}\left(f^{\bullet}\right): H S_{\mathcal{E}\left(x_{n}\right)}^{*}\left(\mathcal{C}^{\bullet}\right) \rightarrow H S_{\mathcal{E}\left(x_{n}\right)}^{*}\left(\mathcal{D}^{\bullet}\right) .
$$

Proof. For every $p \geqslant 0$ let

$$
L_{\geqslant p}^{*}\left(\mathcal{C}^{\bullet}, \mathcal{E}\left(x_{n}\right)\right)=\left\{\left(\varphi_{n}\right)_{n \geqslant p} \in \prod_{m=p}^{\infty} C^{m} \mid\left(\left\|\varphi_{n}\right\|\right)_{n \geqslant p} \in \mathcal{E}\left(x_{n}\right)\right\} .
$$

Then we have a short exact sequence of complexes of the form

$$
0 \rightarrow L_{\geqslant p}^{*}\left(\mathcal{C}^{\bullet}, \mathcal{E}\left(x_{n}\right)\right) \rightarrow \operatorname{Tot}_{\mathcal{E}\left(x_{n}\right)}^{*}\left(\mathcal{C}^{\bullet}\right) \rightarrow L_{<p}^{*}\left(\mathcal{C}^{\bullet}\right) \rightarrow 0
$$

where

$$
L_{<p}^{*}\left(\mathcal{C}^{\bullet}\right)=\left\{\left(\varphi_{n}\right)_{n \geqslant 0} \in \prod_{m=0}^{\infty} C^{m} \mid \varphi_{n}=0 \text { for every } n \geqslant p\right\} .
$$

Notice that the subcomplex $L_{<p}^{*}\left(\mathcal{C}^{\bullet}\right)$ is the same for every sequence $\left(x_{n}\right)_{n \geqslant 0}$, and therefore, is independent of the given sequence. Since $L_{<p}^{*}\left(\mathcal{C}^{\bullet}\right)$ is a bounded double complex whose rows are exact, it is acyclic. In other words, there is a natural quasi-isomorphism of the form

$$
\lim _{\longleftarrow p} L_{\geqslant p}^{*}\left(\mathcal{C}^{\bullet}, \mathcal{E}\left(x_{n}\right)\right) \rightarrow \operatorname{Tot}_{\mathcal{E}\left(x_{n}\right)}^{*}\left(\mathcal{C}^{\bullet}\right) .
$$

PROPOSITION 3.6. If $H S_{\mathcal{E}\left(x_{n}\right)}^{*}$ is a cohomological $\delta$-functor then $H S_{\mathcal{E}\left(x_{n}\right)}^{*}$ must be trivial on the subcategory of bounded cocyclic objects.

Proof. By definition, bounded objects are stably isomorphic to the ground field.

REMARK 3.7. We observe that the well-behaved asymptotic analogues of the $(b, B)$ and the cyclic bicomplexes diverge substantially: the former collapses onto the algebraic periodic cohomology as shown in Proposition 3.3 while the latter becomes trivial as shown in Proposition 3.5 on the subcategory of bounded cocyclic objects.

REMARK 3.8. Recall that in passing from the ordinary cyclic cohomology to the periodic cyclic cohomology, we replace the group cohomologies of cyclic groups with the Tate cohomology of the cyclic groups along the rows. The net effect is that we kill zero-divisors in the group cohomology of cyclic groups, or equivalently, we use a cohomology theory which is stable in that direction using the periodicity operator $S$. Now, as we show in Proposition 3.5. we need a cohomology theory which is stable also in the Hochschild direction. Moreover, we also see that all such well-behaved asymptotic cohomologies of cocyclic objects are determined up to stable isomorphisms, as opposed to ordinary quasi-isomorphisms of cocyclic objects. 


\section{Products in asymptotic cyclic cohomology}

4.1. Products of (co)cyclic modules, and mixed complexes. The category of cocyclic objects is a strict monoidal category with the monoidal product of two cocyclic modules defined diagonally. Namely, if $\mathcal{C}^{\bullet}$ and $\mathcal{D}^{\bullet}$ are two cocyclic modules, their product $\operatorname{Diag}\left(\mathcal{C}^{\bullet} \widehat{\otimes} \mathcal{D}^{\bullet}\right)$ is defined to be the graded module $\bigoplus_{n \in \mathbb{N}} \mathcal{C}^{n} \widehat{\otimes} \mathcal{D}^{n}$ together with

$$
\begin{aligned}
& d_{i}^{\mathcal{C}} \widehat{\otimes} d_{i}^{\mathcal{D}}: \mathcal{C}^{n} \widehat{\otimes} \mathcal{D}^{n} \longrightarrow \mathcal{C}^{n+1} \widehat{\otimes} \mathcal{D}^{n+1}, \quad 0 \leqslant i \leqslant n+1, \\
& s_{j}^{\mathcal{C}} \widehat{\otimes} s_{j}^{\mathcal{D}}: \mathcal{C}^{n} \widehat{\otimes} \mathcal{D}^{n} \longrightarrow \mathcal{C}^{n-1} \widehat{\otimes} \mathcal{D}^{n-1}, \quad 0 \leqslant j \leqslant n-1, \\
& t_{n}^{\mathcal{C}} \widehat{\otimes} t_{n}^{\mathcal{D}}: \mathcal{C}^{n} \widehat{\otimes} \mathcal{D}^{n} \longrightarrow \mathcal{C}^{n} \widehat{\otimes} \mathcal{D}^{n} \text {. }
\end{aligned}
$$

Similarly, the category of mixed complexes has their own strict monoidal product defined as follows: Given two mixed complexes $\left(\mathcal{C}^{*}, b_{*}^{\mathcal{C}}, B_{*}^{\mathcal{C}}\right)$ and $\left(\mathcal{D}^{*}, b_{*}^{\mathcal{D}}, B_{*}^{\mathcal{D}}\right)$, the product complex is the graded product of these mixed complexes

$$
(\mathcal{C} \widehat{\otimes} \mathcal{D})^{n}=\bigoplus_{p+q=n} \mathcal{C}^{p} \widehat{\otimes} \mathcal{D}^{q}
$$

together with the differentials

$$
b_{n}=\sum_{p+q=n}\left(b_{p}^{\mathcal{C}} \widehat{\otimes} \mathrm{id}_{q}\right)+(-1)^{p}\left(\mathrm{id}_{p} \widehat{\otimes} b_{q}^{\mathcal{D}}\right), \quad B_{n}=\sum_{p+q=n}\left(B_{p}^{\mathcal{C}} \widehat{\otimes} \mathrm{id}_{q}\right)+(-1)^{p}\left(\mathrm{id}_{p} \widehat{\otimes} B_{q}^{\mathcal{D}}\right) .
$$

We shall denote the first summands by $\vec{b}$ and $\vec{B}$, and the second summands by $\uparrow b$ and $\uparrow B$, respectively.

4.2. Cup product in asymptotic cyclic cohomology. The functor that sends a cocyclic object $\mathcal{C}^{\bullet}$ to its mixed complex $\mathcal{M}\left(\mathcal{C}^{\bullet}\right)$ is weakly monoidal. In other words, for every pair of cocyclic objects $\mathcal{C}^{\bullet}$ and $\mathcal{D}^{\bullet}$, there are natural quasi-isomorphisms of the form

$$
\operatorname{Sh}_{\mathcal{C} \bullet, \mathcal{D} \bullet}: \mathcal{M}\left(\operatorname{Diag}\left(\mathcal{C}^{\bullet} \widehat{\otimes} \mathcal{D}^{\bullet}\right)\right) \rightarrow \mathcal{M}\left(\mathcal{C}^{\bullet}\right) \widehat{\otimes} \mathcal{M}\left(\mathcal{D}^{\bullet}\right)
$$

implemented by the cyclic shuffle maps. This follows from the cyclic Eilenberg-Zilber Theorem. See [18, Theorem 4.3.8], [9], 15], or [17].

Next, we show that the functor which sends a cocyclic Banach module to an asymptotic complex is weakly monoidal in the following sense.

Proposition 4.1. Assume $\mathcal{C}^{\bullet}$ and $\mathcal{D}^{\bullet}$ are two cocyclic objects, and let $\left(x_{n}\right)_{n \geqslant 0}$ and $\left(y_{n}\right)_{n \geqslant 0}$ be two non-decreasing sequences of positive real numbers. Then there is a natural morphism of differential graded $\mathbb{C}$-vector spaces of the form

$$
C_{\mathcal{E}\left(x_{n}\right)}^{*}\left(\mathcal{C}^{\bullet}\right) \widehat{\otimes} C_{\mathcal{E}\left(y_{n}\right)}^{*}\left(\mathcal{D}^{\bullet}\right) \longrightarrow C_{\mathcal{E}\left(x_{n} y_{n}\right)}^{*}\left(\operatorname{Diag}\left(\mathcal{C}^{\bullet} \widehat{\otimes} \mathcal{D}^{\bullet}\right)\right)
$$

Proof. The given map is the usual external cup product in cyclic cohomology. Thus its compatibility with the differential maps is immediate. So, we only need to show that it does land in the right subcomplex in the target. To this end we observe that

$$
\mathbf{u}_{k} \cup \mathbf{v}_{n-k}=\sum_{\mu \in \operatorname{Sh}(\mathrm{k}, \mathrm{n}-\mathrm{k})} d_{\bar{\mu}(n)} \ldots d_{\bar{\mu}(k+1)} \mathbf{u}_{k} \otimes d_{\bar{\mu}(k)} \ldots d_{\bar{\mu}(1)} \mathbf{v}_{n-k}
$$

and that

$$
\left\|\sum_{k=0}^{n} \mathbf{u}_{k} \cup \mathbf{v}_{n-k}\right\| \leqslant \sum_{k=0}^{n}\left\|\mathbf{u}_{k} \cup \mathbf{v}_{n-k}\right\| \leqslant \sum_{k=0}^{n}\left(\begin{array}{l}
n \\
k
\end{array}\right)\left\|\mathbf{u}_{k}\right\|\left\|\mathbf{v}_{n-k}\right\| .
$$


The second inequality follows from the normalization of the cyclic objects. As a result, we get

$$
\frac{\left\|\sum_{k=0}^{m} \mathbf{u}_{k} \cup \mathbf{v}_{m-k}\right\| r^{m}}{x_{m} y_{m}} \leqslant \sum_{k=0}^{m}\left(\begin{array}{c}
m \\
k
\end{array}\right) \frac{\left\|\mathbf{u}_{k}\right\|\left\|\mathbf{v}_{m-k}\right\| r^{m}}{x_{m} y_{m}} \leqslant \sum_{k=0}^{m} \frac{\left\|\mathbf{u}_{k}\right\|\left\|\mathbf{v}_{m-k}\right\|(2 r)^{m}}{x_{m} y_{m}}
$$

On the other hand, since the sequences $\left(x_{n}\right)_{n \geqslant 0}$ and $\left(y_{n}\right)_{n \geqslant 0}$ are non-decreasing,

$$
x_{k} y_{n-k} \leqslant x_{n} y_{n}, \quad 0 \leqslant k \leqslant n,
$$

thus we get

$$
\frac{\left\|\sum_{k=0}^{m} \mathbf{u}_{k} \cup \mathbf{v}_{m-k}\right\| r^{m}}{x_{m} y_{m}} \leqslant \sum_{k=0}^{m} \frac{\left\|\mathbf{u}_{k}\right\|\left\|\mathbf{v}_{m-k}\right\|(2 r)^{m}}{x_{k} y_{m-k}}
$$

In total, we have

$$
\sup _{m \geqslant n} \frac{\left\|\sum_{k=0}^{m} \mathbf{u}_{k} \cup \mathbf{v}_{m-k}\right\| r^{m}}{x_{m} y_{m}} \leqslant \sup _{m \geqslant n} \sum_{k=0}^{m} \frac{\left\|\mathbf{u}_{k}\right\|(\sqrt{2} r)^{k}}{x_{k}} \cdot \sup _{m \geqslant n} \sum_{k=0}^{m} \frac{\left\|\mathbf{v}_{k}\right\|(\sqrt{2} r)^{k}}{y_{k}},
$$

from which the claim follows by applying the limit $n \rightarrow \infty$.

4.3. Cup product in asymptotic Hopf-cyclic cohomology. Let $\mathcal{B}$ be a (Banach-) Hopf algebra with a fixed MPI $(\delta, \sigma)$, and let $C$ be a Banach $\mathcal{B}$-module coalgebra. In other words,

$$
\Delta(h(c))=h_{(1)}\left(c_{(1)}\right) h_{(2)}\left(c_{(2)}\right), \quad \varepsilon(h(c))=\varepsilon(h) \varepsilon(c), \quad\|h(c)\| \leqslant\|h\|\|c\|,
$$

for every $h \in \mathcal{B}$ and $c \in C$. Now, assume $A$ is a Banach $\mathcal{B}$-module algebra which means that we have

$$
h(a b)=h_{(1)}(a) h_{(2)}(b), \quad h(1)=\varepsilon(h) 1, \quad\|h(a)\| \leqslant\|h\|\|a\|,
$$

for any $h \in \mathcal{B}$, and any $a, b \in A$. We are going to say that $A$ admits an $\mathcal{B}$-equivariant action of $C$ if there is a map $C \otimes A \rightarrow A$ satisfying

$$
c(a b)=c_{(1)}(a) c_{(2)}(b),\left(S^{-1}(h)(c)\right)(a)=c(h(a)), c(1)=\varepsilon(c) 1,\|c(a)\| \leqslant\|c\|\|a\|
$$

for any $c \in C$, and any $a, b \in A$. Now, let

(i) $\mathcal{C}_{\mathcal{B}}^{\bullet}(A ; \delta, \sigma)$ be the standard Hopf-cocyclic object associated with the $\mathcal{B}$-module algebra $A$ with coefficients in the $\operatorname{MPI}(\delta, \sigma)$,

(ii) $\mathcal{C}_{\mathcal{B}}^{\bullet}(C ; \delta, \sigma)$ be the standard Hopf-cocyclic object associated with the $\mathcal{B}$-module coalgebra $C$ with coefficients in the $\operatorname{MPI}(\delta, \sigma)$,

(iii) and $\mathcal{C}^{\bullet}(A)$ be the standard cocyclic object associated with the algebra $A$.

The proof of the following lemma is a straightforward but tedious check of the compatibility conditions between the cocyclic structure maps, and therefore is omitted.

LEMmA 4.2. If $A$ admits a $\mathcal{B}$-equivariant action of $C$, then there is a well-defined morphism of cocyclic objects of the form

$$
\Gamma^{\bullet}: \operatorname{Diag}\left(\mathcal{C}_{\mathcal{B}}^{\bullet}(C ; \delta, \sigma) \widehat{\otimes} \mathcal{C}_{\mathcal{B}}^{\bullet}(A ; \delta, \sigma)\right) \rightarrow \mathcal{C}^{\bullet}(A)
$$

where the range is the standard cocyclic object associated with an algebra $A$ where we define

$$
\Gamma^{n}\left(c^{0} \otimes \cdots \otimes c^{n} \mid \varphi\right)\left(a_{0} \otimes \cdots \otimes a_{n}\right)=\varphi\left(c^{0}\left(a_{0}\right), \ldots, c^{n}\left(a_{n}\right)\right)
$$

for every $c^{0} \otimes \cdots \otimes c^{n} \in \mathcal{C}_{\mathcal{B}}^{n}(C ; \delta, \sigma)$ and $\varphi \in \mathcal{C}_{\mathcal{B}}^{n}(A ; \delta, \sigma)$. 
Using Lemma 4.2 in combination with Proposition 4.1, we get the following.

ThEOREM 4.3. Let $\mathcal{B}$ be a (Banach-)Hopf algebra with an $\operatorname{MPI}(\delta, \sigma), A$ a unital Banach $\mathcal{B}$-module algebra, and $C$ a Banach $\mathcal{B}$-module coalgebra such that $A$ admits a $\mathcal{B}$-equivariant action of $C$. Assume also that $\left(x_{n}\right)_{n \geqslant 0}$ and $\left(y_{n}\right)_{n \geqslant 0}$ are two non-decreasing sequences in $(0, \infty)$. Then there is a cup product of the form

$$
\cup: H P_{\mathcal{B}, \mathcal{E}\left(x_{n}\right)}^{i}(C) \otimes H P_{\mathcal{B}, \mathcal{E}\left(y_{n}\right)}^{j}(A) \rightarrow H P_{\mathcal{E}\left(x_{n} y_{n}\right)}^{i+j}(A)
$$

where $H P_{\mathcal{B}, \mathcal{E}\left(x_{n}\right)}^{i}(C)$ and $H P_{\mathcal{B}, \mathcal{E}\left(x_{n}\right)}^{i}(A)$ respectively denote the asymptotic Hopf-cyclic cohomologies of $C$ and $A$, while $H P_{\mathcal{E}\left(x_{n}\right)}^{i}(A)$ denotes the asymptotic cyclic cohomology of $A$.

5. Asymptotic cyclic cohomology of the simplex. Let $\Delta^{n}$ denote the geometric $n$-simplex, and let

$$
\Delta^{\bullet}:=\bigoplus_{n \geqslant 0} \mathbb{C}\left[\Delta^{n}\right]
$$

In this section we are going to introduce a non-trivial cocycle in the $\mathcal{E}(1)$-asymptotic cyclic cohomology of the cocyclic module (5.1).

We recall from [9] that the geometric $n$-simplex can be described in two different coordinate systems

$$
\left(t_{0}, \ldots, t_{n+1}\right) \quad \text { where } \quad 0=t_{0} \leqslant t_{1} \leqslant \ldots \leqslant t_{n} \leqslant t_{n+1}=1,
$$

and

$$
\left(t_{0}, \ldots, t_{n}\right) \quad \text { where } t_{i} \in[0,1] \text { and } 1=\sum_{i=0}^{n} t_{i} .
$$

Notice that the 0 -simplex contains one single point. In the first coordinate system this is represented by the sequence $(0,1)$ while in the second it is simply 1 . We will denote this point by $*$ independent of the coordinate system chosen. We will prefer the first coordinate system for our calculations below. Also, in writing an element $\left(t_{0}, \ldots, t_{n+1}\right)$ we will drop $t_{0}=0$ and $t_{n+1}=1$ from the coordinates for convenience.

We leave the proof of the following fact to the reader:

LEMma 5.1. The graded space (5.1) has a cocyclic structure determined by the coface operators $\delta_{i}^{n}: \Delta^{n} \longrightarrow \Delta^{n+1}$

$$
\delta_{i}\left(t_{1}, \ldots, t_{n}\right)= \begin{cases}\left(0, t_{1}, \ldots, t_{n}\right) & \text { if } i=0, \\ \left(t_{1}, \ldots, t_{i}, t_{i}, \ldots, t_{n}\right) & \text { if } 0 \leqslant i \leqslant n, \\ \left(t_{1}, \ldots, t_{n}, 1\right) & \text { if } i=n+1,\end{cases}
$$

the codegeneracy operators $\sigma_{j}^{n}: \delta^{n} \longrightarrow \delta^{n-1}$

$$
\sigma_{j}^{n}\left(t_{1}, \ldots, t_{n}\right)=\left(t_{1}, \ldots, \widehat{t_{j+1}}, \ldots, t_{n}\right),
$$

and the cyclic operators $\tau_{n}: \Delta^{n} \longrightarrow \Delta^{n}$

$$
\tau_{n}\left(t_{1}, \ldots, t_{n}\right)=\left(t_{2}-t_{1}, t_{3}-t_{1}, \ldots, t_{n}-t_{1}, 1-t_{1}\right),
$$

defined for $0 \leqslant i \leqslant n+1$ and $0 \leqslant j \leqslant n-1$. 
Given any sequence of positive numbers $\left(x_{n}\right)_{n \geqslant 0}$, in the construction of the asymptotic complex

$$
\cdots \longrightarrow C_{\mathcal{E}\left(x_{n}\right)}^{0}\left(\Delta^{\bullet}\right) \stackrel{b+B}{\longrightarrow} C_{\mathcal{E}\left(x_{n}\right)}^{1}\left(\Delta^{\bullet}\right) \stackrel{b+B}{\longrightarrow} C_{\mathcal{E}\left(x_{n}\right)}^{0}\left(\Delta^{\bullet}\right) \longrightarrow \cdots
$$

we endow the $n$-simplex $\Delta^{n}$ with the norm $\left\|\left(t_{1}, \ldots, t_{n}\right)\right\|:=\max \left\{t_{i} \mid i=1, \ldots, n\right\}$.

Proposition 5.2. The cochain $\varphi=\left(\varphi_{2 n}\right)_{n \geqslant 0} \in C_{\infty}^{0}\left(\Delta^{\bullet}\right)$ of infinite support, given by

$$
\varphi_{2 n}= \begin{cases}* & \text { if } n=0 \\ \frac{(-1)^{n}}{2^{n} n !} \sum_{r=0}^{n} \tau_{2 n}^{2 r} \delta_{0}^{2 n}(*) & \text { if } n \geqslant 1\end{cases}
$$

is a non-trivial $\mathcal{E}(1)$-asymptotic cocycle.

Proof. Let us first note that

$$
\sqrt[n]{\left\|\varphi_{2 n}\right\|} \leqslant \sqrt[n]{\frac{n+1}{2^{n} n !}}=\frac{\sqrt[n]{n+1}}{2 \sqrt[n]{n !}}
$$

Then from Stirling's approximation we get

$$
\limsup _{n \rightarrow \infty}\left\|\varphi_{2 n}\right\|=0
$$

which implies $\left(\varphi_{2 n}\right)_{n \geqslant 0} \in C_{\mathcal{E}(1)}^{0}\left(\Delta^{\bullet}\right)$.

For $n>1$, we see that

$$
\begin{aligned}
B\left(\delta_{0}^{2 n}(*)\right) & =N_{2 n-1} \sigma_{2 n-1} \tau_{2 n}\left(\operatorname{Id}-\tau_{2 n}\right) \delta_{0}^{2 n}(*) \\
& =N_{2 n-1} \sigma_{2 n-1}((\underbrace{0, \ldots, 0}_{2 n-1 \text { times }}, 1)-(\underbrace{0, \ldots, 0}_{2 n-2 \text { times }}, 1,1)) \\
& =\left(\operatorname{Id}-\tau_{2 n-1}+\tau_{2 n-1}^{2}-\ldots-\tau_{2 n-1}^{2 n-1}\right)((\underbrace{0, \ldots, 0}_{2 n-1 \text { times }})-(\underbrace{0, \ldots, 0}_{2 n-2 \text { times }}, 1)) \\
& =\left(\operatorname{Id}-\tau_{2 n-1}+\tau_{2 n-1}^{2}-\ldots-\tau_{2 n-1}^{2 n-1}\right)\left(\operatorname{Id}-\tau_{2 n-1}\right) \delta_{0}^{2 n-1}(*)=2 N_{2 n-1} \delta_{0}^{2 n-1}(*) .
\end{aligned}
$$

Similarly, we get

$$
\begin{aligned}
B\left(\tau_{2 n}^{2 r} \delta_{0}^{2 n}(*)\right) & =N_{2 n-1} \sigma_{2 n-1} \tau_{2 n}\left(\operatorname{Id}-\tau_{2 n}\right) \tau_{2 n}^{2 r} \delta_{0}^{2 n}(*) \\
& =N_{2 n-1}\left(\tau_{2 n-1}^{2 r}-\tau_{2 n-1}^{2 r+1}\right) \delta_{0}^{2 n-1}(*)=2 N_{2 n-1} \delta_{0}^{2 n-1}(*),
\end{aligned}
$$

and

$$
\begin{aligned}
B\left(\tau_{2 n}^{2 n} \delta_{0}^{2 n}(*)\right) & =N_{2 n-1} \sigma_{2 n-1} \tau_{2 n}\left(\mathrm{Id}-\tau_{2 n}\right) \tau_{2 n}^{2 n} \delta_{0}^{2 n}(*) \\
& =N_{2 n-1} \sigma_{2 n-1}\left(\mathrm{Id}-\tau_{2 n}\right) \delta_{0}^{2 n}(*)=N_{2 n-1}\left(\mathrm{Id}-\tau_{2 n}\right) \delta_{0}^{2 n}(*)=0
\end{aligned}
$$

On the other hand, we have

$$
b\left(\tau_{2 n}^{2 r} \delta_{0}^{2 n}(*)\right)=\tau_{2 n}^{2 r} \delta_{0}^{2 n}(*)-\tau_{2 n}^{2 r-1} \delta_{0}^{2 n}(*) .
$$


As a result, we see the following picture:

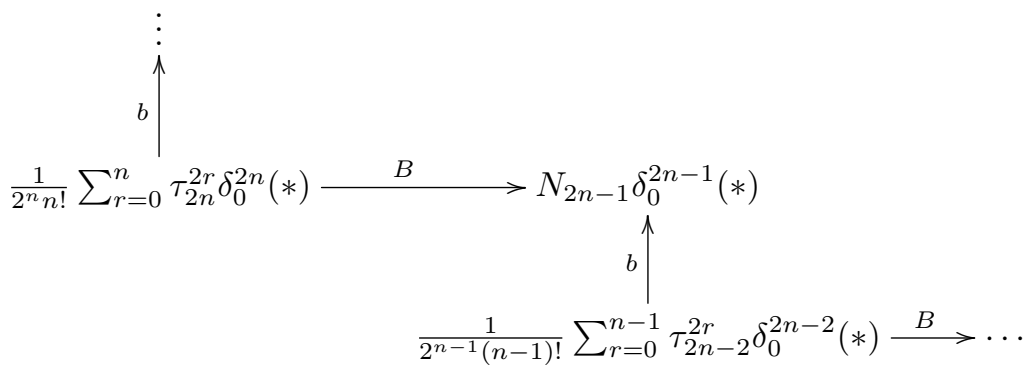

As for the non-triviality, we observe that for any $0 \leqslant s \leqslant 2 n+1$,

$$
\begin{aligned}
B_{2 n+1} \tau_{2 n}^{s} \delta_{0}^{2 n+1}(*) & =N_{2 n} \sigma_{2 n} \tau_{2 n}\left(\operatorname{Id}+\tau_{2 n}\right) \tau_{2 n}^{s} \delta_{0}^{2 n+1}(*) \\
& =N_{2 n}\left(\tau_{2 n}^{s}+\tau_{2 n}^{s+1}\right) \delta_{0}^{2 n+1}(*)=2 N_{2 n} \delta_{0}^{2 n+1}(*),
\end{aligned}
$$

whereas

$$
b_{2 n-1} \tau_{2 n}^{s} \delta_{0}^{2 n-1}(*)=\tau_{2 n}^{s} \delta_{0}^{2 n-1}(*)= \begin{cases}\tau_{2 n}^{s+1} \delta_{0}^{2 n}(*), & \text { if } s \text { is even, } \\ \tau_{2 n}^{s} \delta_{0}^{2 n}(*), & \text { if } s \text { is odd }\end{cases}
$$

The claim then follows.

REMARK 5.3. Note that the cocycle $\left(\varphi_{2 n}\right)_{n \geqslant 0} \in C_{\mathcal{E}(1)}^{0}\left(\Delta^{\bullet}\right)$ is not entire, that is, $\left(\varphi_{2 n}\right)_{n \geqslant 0}$ is not in $C_{\varepsilon}^{0}\left(\Delta^{\bullet}\right)$. Indeed,

$$
\sum_{n \geqslant 0} \frac{(2 n) !\left\|\varphi_{2 n}\right\|}{n !}|z|^{n} \geqslant \sum_{n \geqslant 0} \frac{(2 n) !}{2^{n} n ! n !}|z|^{n},
$$

where the latter has radius of convergence $\frac{1}{2}$.

\section{Asymptotic characteristic map and the index cocycles}

6.1. Theta-summable Fredholm modules and the Chern character. We are going to recall the construction of the Chern character formula of a theta-summable Fredholm module from [12, 10], see also [2].

Definition 6.1. A theta-summable Fredholm module over a unital Banach algebra $\mathcal{A}$ is a pair $(\mathcal{H}, \not D)$ consisting of a $\mathbb{Z}_{2}$-graded Hilbert space $\mathcal{H}=\mathcal{H}^{+} \oplus \mathcal{H}^{-}$, admitting a continuous representation of $\mathcal{A}$, and an odd self-adjoint operator $\not D: \mathcal{H}^{ \pm} \rightarrow \mathcal{H}^{\mp}$, such that

(i) for any $a \in \mathcal{A}$, the operator $[\not D, a]$ is densely defined, extends to a bounded operator on $\mathcal{H}$, and there is $N(\not D)>0$ with $\|a\|+\|[\not D, a]\| \leqslant N(\not D)\|a\|$,

(ii) $\operatorname{Tr} e^{-(1-\varepsilon) \not D^{2}}$ is finite for some $\varepsilon>0$.

In order to define the Chern character of a Fredholm module, let $e(t):=e^{-t \not D^{2}}$, and

$$
\begin{aligned}
\left\langle a_{0}, \ldots,\right. & \left.a_{n}\right\rangle_{n} \\
& :=\int_{\Delta^{n}} \operatorname{Str}\left(a_{0} e\left(t_{1}\right) a_{1} e\left(t_{2}-t_{1}\right) a_{2} \ldots e\left(t_{n}-t_{n-1}\right) a_{n} e\left(1-t_{n}\right)\right) d t_{1} \ldots d t_{n},
\end{aligned}
$$


where $\Delta^{n}$ denotes the $n$-simplex given by $\left(t_{1}, \ldots, t_{n}\right)$ with $0 \leqslant t_{1} \leqslant \ldots \leqslant t_{n} \leqslant 1$, and $\operatorname{Str}(a):=\operatorname{Tr}\left(\left.a\right|_{\mathcal{H}^{+}}\right)-\operatorname{Tr}\left(\left.a\right|_{\mathcal{H}^{-}}\right)$is the supertrace of an operator $a: \mathcal{H} \rightarrow \mathcal{H}$.

The even entire cochain $\operatorname{Ch}(\not D) \in C_{\varepsilon}^{0}(\mathcal{A})$ given by

$$
\mathrm{Ch}^{2 n}(\not D)\left(a_{0}, \ldots, a_{2 n}\right):=\left\langle a_{0},\left[\not D, a_{1}\right], \ldots,\left[\not D, a_{2 n}\right]\right\rangle_{2 n}, \quad n \geqslant 0
$$

is called the Chern character of a theta-summable Fredholm module $(\mathcal{H}, \not D)$ over a unital Banach algebra $\mathcal{A}$. It is proved in [12] that $\left[\left(\mathrm{Ch}^{2 n}(\not D)\right)_{n \geqslant 0}\right] \in H P_{\varepsilon}^{0}(\mathcal{A})$ represents a nontrivial even entire class. This cocycle is usually referred as the JLO-cocycle in the literature.

Following [8], we recall also the odd theta-summable Fredholm module.

Definition 6.2. Given a Banach $*$-algebra $\mathcal{A}$, an odd theta-summable Fredholm module is also a pair $(\mathcal{H}, \not D)$ consisting of a Hilbert space $\mathcal{H}$ as a continuous $*$-representation of $\mathcal{A}$, and a self-adjoint operator $\not D: \mathcal{H} \rightarrow \mathcal{H}$ so that

(i) there is $c>0$ such that $\|[\not D, a]\| \leqslant c\|a\|$, for all $a \in \mathcal{A}$,

(ii) if $t>0$, then $\operatorname{Tr} e^{-t \not D^{2}}$ is finite.

Given two self-adjoint operators $A_{0}$ and $A_{1}$ on $\mathcal{H}$, the integer $\operatorname{sf}\left(A_{0}, A_{1}\right)$, called the spectral flow from $A_{0}$ to $A_{1}$, is introduced in [1, Section 7]. In particular, for an odd theta-summable Fredholm module $(\mathcal{H}, \not D)$ on $\mathcal{A}$, and a unitary $g \in U_{N}(\mathcal{A})$, the spectral flow defines a pairing

$$
K^{1}(\mathcal{A}) \times K_{1}(\mathcal{A}) \longrightarrow \mathbb{Z}, \quad\langle D, g\rangle:=\operatorname{sf}\left(D, g^{-1} D g\right)
$$

between the $K$-theory and the $K$-homology of $\mathcal{A}$. Furthermore, it is shown in [8, Section 2] that

$$
\operatorname{sf}\left(D, g^{-1} D g\right)=\frac{1}{\sqrt{\pi}} \int_{0}^{1} \operatorname{Tr}\left(\dot{D}_{u} e^{-\not D_{u}^{2}}\right) d u,
$$

where $\not D_{u}:=(1-u) \not D+u g^{-1} \not D g$, and $\dot{D}_{u}:=g^{-1}[\not D, g]$.

6.2. JLO-cocycle revisited. Let $\mathbb{C}[\mathbb{R}]$ be the polynomial commutative and cocommutative Hopf algebra generated by $e(t)$ where $t \in \mathbb{R}$ subject to the relations

$$
e(t) e(s)=e(t+s) \quad \text { and } \quad \Delta(e(t))=e(t) \otimes e(t)
$$

for every $t, s \in \mathbb{R}$. We set $1=e(0)$. This is the group ring $\mathbb{C}[\mathbb{R}]$ of the group $(\mathbb{R},+, 0)$.

Let $\mathcal{Z}$ be the two-dimensional coalgebra $\langle 1, D\rangle$, where 1 is group-like and $D$ is primitive. Consider the operator $\varphi_{n}: \mathcal{A}^{\otimes n+1} \rightarrow \operatorname{Hom}\left(\mathcal{Z}^{\otimes n+1} \times \Delta^{n}, \mathbb{C}\right)$ defined by

$$
\begin{aligned}
\varphi_{n}\left(a_{0} \otimes \cdots a_{n}\right) & \left(D^{\epsilon_{0}} \otimes \cdots \otimes D^{\epsilon_{n}} \mid t_{0}, \ldots, t_{n}\right) \\
= & \operatorname{Str}\left(a_{0} e^{-t_{0} \not D^{\epsilon_{0}}}\left[\not D, a_{1}\right] e^{-t_{1} \not D^{\epsilon_{1}}} \cdots\left[\not D, a_{n}\right] e^{-t_{n} \not D^{\epsilon_{n}}}\right),
\end{aligned}
$$

and let us restrict the arguments in $\mathbb{C}[\mathbb{R}]^{\otimes n+1}$ to $\Delta^{n}$. In the next step we split the space of continuous functionals $\operatorname{Hom}\left(\mathcal{Z}^{\otimes n+1} \times \Delta^{n}, \mathbb{C}\right)$ as $\operatorname{Hom}\left(\mathcal{Z}^{\otimes n+1}, \mathbb{C}\right) \widehat{\otimes} \operatorname{Hom}\left(\Delta^{n}, \mathbb{C}\right)$. Using the restriction function rest : $\left.\gamma_{n} \mapsto \gamma_{n}\right|_{1 \otimes \cdots \otimes 1}$ on $\operatorname{Hom}\left(\mathcal{Z}^{\otimes n+1}, \mathbb{C}\right)$, and the integral on $\operatorname{Hom}\left(\Delta^{n}, \mathbb{C}\right)$, we get a trace on the product. The JLO-cocycle is the composition

$$
\mathcal{A}^{\otimes n+1} \stackrel{\varphi_{n}}{\longrightarrow} \operatorname{Hom}\left(\mathcal{Z}^{\otimes n+1} \times \Delta^{n}, \mathbb{C}\right) \stackrel{\cong}{\longrightarrow} \operatorname{Hom}\left(\mathcal{Z}^{\otimes n+1}, \mathbb{C}\right) \widehat{\otimes} \operatorname{Hom}\left(\Delta^{n}, \mathbb{C}\right) \stackrel{\text { rest } \otimes \int}{\longrightarrow} \mathbb{C} .
$$


In the next subsections we are going to construct a new cocycle in the image of a Connes-Moscovici type characteristic map which behaves in the same way as the JLOcocycle in terms of the Chern pairing with the K-theory.

6.3. Characteristic map. In this subsection we are going to construct a characteristic map from the asymptotic cyclic cohomology of the simplex we calculated in Subsection 5 to the entire cohomology of the algebra $\mathcal{A}$. Then we are going to observe that the image of the cocycle 5.4 yields the index of the theta-summable Fredholm module $(\mathcal{H}, \not D)$ after paired with the K-theory.

Proposition 6.3. Let $\mathcal{A}$ be a $\mathcal{B}$-module algebra for a Hopf-algebra $\mathcal{B}$ with the modular pair in involution $(\varepsilon, 1)$. Assume also that $\mathcal{B}$ acts on $e(t)$ trivially, for all $t \geqslant 0$, and the supertrace $\operatorname{Str}$ is invariant under the $\mathcal{B}$-action. There is a characteristic homomorphism of cocyclic objects given by $\chi: \operatorname{Diag}^{\bullet}\left(C_{\mathcal{B}}^{\bullet}(\mathcal{B} ; \epsilon, 1) \otimes \Delta^{\bullet}\right) \longrightarrow C^{\bullet}(\mathcal{A})$

$$
\begin{aligned}
\chi\left(h^{1} \otimes \cdots \otimes h^{n} \mid t_{1}, \ldots, t_{n}\right)\left(a_{0}, \ldots, a_{n}\right) \\
:=\operatorname{Str}\left(a_{0} e\left(t_{1}\right) h^{1}\left(a_{1}\right) e\left(t_{2}-t_{1}\right) \ldots e\left(t_{n}-t_{n-1}\right) h^{n}\left(a_{n}\right) e\left(1-t_{n}\right)\right),
\end{aligned}
$$

where $C_{\mathcal{B}}^{\bullet}(\mathcal{B} ; \epsilon, 1)$ is the standard Hopf cocyclic object associated to $\mathcal{B}$ with coefficients in the $\operatorname{MPI}(\epsilon, 1)$.

Proof. We are going to see the compatibility of the characteristic homomorphism with the cocyclic structure. To begin with,

$$
\begin{aligned}
& \chi\left(d_{0}\left(h^{1} \otimes \cdots \otimes h^{n}\right) \mid \delta_{0}\left(t_{1}, \ldots, t_{n}\right)\right)\left(a_{0}, \ldots, a_{n+1}\right) \\
& =\chi\left(1 \otimes h^{1} \otimes \cdots \otimes h^{n} \mid 0, t_{1}, \ldots, t_{n}\right)\left(a_{0}, \ldots, a_{n+1}\right) \\
& =\operatorname{Str}\left(a_{0} a_{1} e\left(t_{1}\right) h^{1}\left(a_{2}\right) e\left(t_{2}-t_{1}\right) \cdots h^{n}\left(a_{n+1}\right) e\left(1-t_{n}\right)\right) \\
& =d_{0} \chi\left(h^{1} \otimes \cdots \otimes h^{n} \mid t_{1}, \ldots, t_{n}\right)\left(a_{0}, \ldots, a_{n+1}\right) .
\end{aligned}
$$

Similarly, for $1 \leqslant i \leqslant n$, we have

$$
\begin{aligned}
& \chi\left(d_{i}\left(h^{1} \otimes \cdots \otimes h^{n}\right) \mid \delta_{i}\left(t_{0}, \ldots, t_{n}\right)\right)\left(a_{0}, \ldots, a_{n+1}\right) \\
& =\chi\left(h^{1} \otimes \cdots \otimes h_{(1)}^{i} \otimes h_{(2)}^{i} \otimes \cdots \otimes h^{n} \mid t_{1}, \ldots, t_{i}, t_{i}, \ldots, t_{n}\right)\left(a_{0}, \ldots, a_{n+1}\right) \\
& =\operatorname{Str}\left(a_{0} e\left(t_{1}\right) h^{1}\left(a_{1}\right) e\left(t_{2}-t_{1}\right) \cdots e\left(t_{i}-t_{i-1}\right) h_{(1)}^{i}\left(a_{i}\right) e\left(t_{i}-t_{i}\right) h_{(2)}^{i}\left(a_{i+1}\right) e\left(t_{i+1}-t_{i}\right)\right. \\
& \left.\quad \cdots h^{n}\left(a_{n+1}\right) e\left(1-t_{n}\right)\right) \\
& =\operatorname{Str}\left(a_{0} e\left(t_{1}\right) h^{1}\left(a_{1}\right) e\left(t_{2}-t_{1}\right) \cdots e\left(t_{i}-t_{i-1}\right) h^{i}\left(a_{i} a_{i+1}\right) e\left(t_{i+1}-t_{i}\right) \cdots h^{n}\left(a_{n+1}\right) e\left(1-t_{n}\right)\right) \\
& =d_{i} \chi\left(h^{1} \otimes \cdots \otimes h^{n} \mid t_{1}, \ldots, t_{n}\right)\left(a_{0}, \ldots, a_{n+1}\right) .
\end{aligned}
$$

For the last coface operator, we have

$$
\begin{aligned}
& \chi\left(d_{n+1}\left(h^{1} \otimes \cdots \otimes h^{n}\right) \mid \delta_{n+1}\left(t_{1}, \ldots, t_{n}\right)\right)\left(a_{0}, \ldots, a_{n+1}\right) \\
& =\chi\left(h^{1} \otimes \cdots \otimes h^{n} \otimes 1 \mid t_{1}, \ldots, t_{n}, 1\right)\left(a_{0}, \ldots, a_{n+1}\right) \\
& =\operatorname{Str}\left(a_{0} e\left(t_{1}\right) h^{1}\left(a_{1}\right) e\left(t_{2}-t_{1}\right) \cdots h^{n}\left(a_{n}\right) e\left(t_{n}-t_{n-1}\right) a_{n+1}\right) \\
& =\operatorname{Str}\left(a_{n+1} a_{0} e\left(t_{1}\right) h^{1}\left(a_{1}\right) e\left(t_{2}-t_{1}\right) \cdots h^{n}\left(a_{n}\right) e\left(t_{n}-t_{n+1}\right)\right) \\
& =d_{n+1} \chi\left(h^{1} \otimes \cdots \otimes h^{n} \mid t_{1}, \ldots, t_{n}\right)\left(a_{0}, \ldots, a_{n+1}\right) .
\end{aligned}
$$


We proceed to the compatibility with the codegeneracies. For $0 \leqslant j \leqslant n-1$,

$$
\begin{aligned}
& \chi\left(s_{j}\left(h^{1} \otimes \cdots \otimes h^{n}\right) \mid \sigma_{j}\left(t_{1}, \ldots, t_{n}\right)\right)\left(a_{0}, \ldots, a_{n+1}\right) \\
& =\chi\left(h^{1} \otimes \cdots \otimes \varepsilon\left(h^{j+1}\right) \otimes \cdots \otimes h^{n} \mid t_{1}, \ldots, t_{j}, t_{j+2}, \ldots, t_{n}\right)\left(a_{0}, \ldots, a_{n-1}\right) \\
& =\operatorname{Str}\left(a_{0} e\left(t_{1}\right) h^{1}\left(a_{1}\right) e\left(t_{2}-t_{1}\right) \cdots h^{j}\left(a_{j}\right) e\left(t_{j+1}-t_{j}\right) h^{j+1}(1) e\left(t_{j+2}-t_{j+1}\right)\right. \\
& \left.\quad \cdot h^{j+2}\left(a_{j+1}\right) \cdots h^{n}\left(a_{n-1}\right) e\left(1-t_{n}\right)\right) \\
& =\chi\left(h^{1} \otimes \cdots \otimes h^{n} \mid t_{1}, \ldots, t_{n}\right)\left(a_{0}, \ldots, a_{j}, 1, \ldots, a_{n-1}\right) \\
& =s_{j} \chi\left(h^{1} \otimes \cdots \otimes h^{n} \mid t_{1}, \ldots, t_{n}\right)\left(a_{0}, \ldots, a_{n-1}\right) .
\end{aligned}
$$

Finally, the compatibility with the cyclic operator goes as follows:

$$
\begin{aligned}
& \chi\left(t_{n}\left(h^{1} \otimes \cdots \otimes h^{n}\right) \mid \tau_{n}\left(t_{1}, \ldots, t_{n}\right)\right)\left(a_{0}, \ldots, a_{n}\right) \\
& =\chi\left(S\left(h^{1}\right) \cdot\left(h^{2} \otimes \cdots \otimes h^{n} \otimes 1\right) \mid t_{2}-t_{1}, \ldots, t_{n}-t_{1}, 1-t_{1}\right)\left(a_{0}, \ldots, a_{n}\right) \\
& =\operatorname{Str}\left(a_{0} e\left(t_{2}-t_{1}\right) S\left(h^{1}\right) \cdot\left(h^{2}\left(a_{1}\right) e\left(t_{3}-t_{2}\right) \cdots h^{n}\left(a_{n-1}\right) e\left(1-t_{n}\right) 1\left(a_{n}\right) e\left(t_{1}\right)\right)\right) \\
& =\operatorname{Str}\left(h^{1}\left(a_{0}\right) e\left(t_{2}-t_{1}\right) h^{2}\left(a_{1}\right) e\left(t_{3}-t_{2}\right) \cdots h^{n}\left(a_{n-1}\right) e\left(1-t_{n}\right) 1\left(a_{n}\right) e\left(t_{1}\right)\right) \\
& =\operatorname{Str}\left(a_{n} e\left(t_{1}\right) h^{1}\left(a_{0}\right) e\left(t_{2}-t_{1}\right) h^{2}\left(a_{1}\right) e\left(t_{3}-t_{2}\right) \cdots h^{n}\left(a_{n-1}\right) e\left(1-t_{n}\right)\right) \\
& =\chi\left(h^{1} \otimes \cdots \otimes h^{n} \mid t_{1}, \ldots, t_{n}\right)\left(a_{n}, a_{0}, \ldots, a_{n-1}\right) \\
& =t_{n} \chi\left(h^{1} \otimes \cdots \otimes h^{n} \mid t_{1}, \ldots, t_{n}\right)\left(a_{0}, \ldots, a_{n}\right),
\end{aligned}
$$

where we used the triviality of the $\mathcal{B}$-action on $e(t)$, and the invariance of Str under the $\mathcal{B}$-action.

6.4. Even index cocycle. We note that, the morphism of cocyclic objects we defined in Proposition 6.3 induces a map on the asymptotic complexes if $\mathcal{B}$ is a Hopf algebra in $\operatorname{Ban}_{\mathbb{C}}$ :

$$
\chi: C_{\mathcal{E}\left(x_{n}\right)}^{i}\left(\operatorname{Diag}\left(C_{\mathcal{B}}^{\bullet}(\mathcal{B} ; \epsilon, 1) \otimes \Delta^{\bullet}\right)\right) \longrightarrow C_{\mathcal{E}\left(x_{n}\right)}^{i}\left(C^{\bullet}(\mathcal{A})\right) .
$$

On the other hand by Proposition 4.1 we have a cup product, and therefore, we get $C_{\mathcal{E}\left(x_{n}\right)}^{i}\left(C_{\mathcal{B}}^{\bullet}(\mathcal{B} ; \epsilon, 1)\right) \otimes C_{\mathcal{E}(1)}^{j}\left(\Delta^{\bullet}\right) \stackrel{\cup}{\longrightarrow} C_{\mathcal{E}\left(x_{n}\right)}^{i+j}\left(\operatorname{Diag}\left(C_{\mathcal{B}}^{\bullet}(\mathcal{B} ; \epsilon, 1) \otimes \Delta^{\bullet}\right)\right) \stackrel{\chi}{\longrightarrow} C_{\mathcal{E}\left(x_{n}\right)}^{i+j}\left(C^{\bullet}(\mathcal{A})\right)$.

We are going to use this setup to define an index cocycle.

Now, let $\mathcal{P}$ be the polynomial Hopf algebra $\mathbb{C}[X]$, and let us define

$$
\mathbf{I}^{0}:=\mathbf{1} \in C^{0}(\mathcal{P}) \quad \text { and } \quad \mathbf{I}^{r}:=\underbrace{\mathbf{I} \cup \ldots \cup \mathbf{I}}_{r \text { times }} \in C^{2 r}(\mathcal{P}),
$$

for any $r \geqslant 1$ where $\mathbf{I}:=1 \otimes 1 \in C^{2}(\mathcal{P})$. For this Hopf algebra we record the following. Proposition 6.4. We have a morphism of differential graded $\mathbb{C}$-vector spaces of the form

$$
\iota: C_{\mathcal{E}(1)}^{*}\left(\Delta^{\bullet}\right) \longrightarrow C_{\mathcal{E}(1)}^{0}\left(C^{\bullet}(\mathcal{P})\right) \widehat{\otimes} C_{\mathcal{E}(1)}^{*}\left(\Delta^{\bullet}\right)
$$

given by

$$
\left(\mathbf{t}_{2 n}\right)_{n \geqslant 0} \mapsto\left(\sum_{r=0}^{n} \alpha_{r}\left(\mathbf{I}^{r} \mid \mathbf{t}_{2 n-2 r}\right)\right)_{n \geqslant 0} \text { and }\left(\mathbf{t}_{2 n+1}\right)_{n \geqslant 0} \mapsto\left(\sum_{r=0}^{n} \alpha_{r}\left(\mathbf{I}^{r} \mid \mathbf{t}_{2 n+1-2 r}\right)\right)_{n \geqslant 0},
$$


where $\left(\alpha_{r}\right)_{r \geqslant 0}$ is a sequence of real numbers defined as

$$
\alpha_{r}= \begin{cases}1 & \text { if } r=0 \\ \frac{1}{(2 r) !}-\frac{1}{(2 r-2) !} & \text { if } r>0 .\end{cases}
$$

Proof. We note that since $\mathbf{I}=b(1)$ we have $b(\mathbf{I})=0$, hence a commutative square of the form

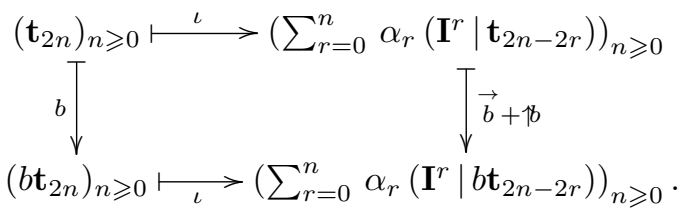

Thus (6.3) commutes with the Hochschild coboundary maps. Similarly, we have

$$
\tau_{2}(\mathbf{I})=S(1) \cdot(1 \otimes 1)=\mathbf{I} .
$$

Therefore, we also have $B_{2}(\mathbf{I})=0$. As a result, we have another commutative square of the form

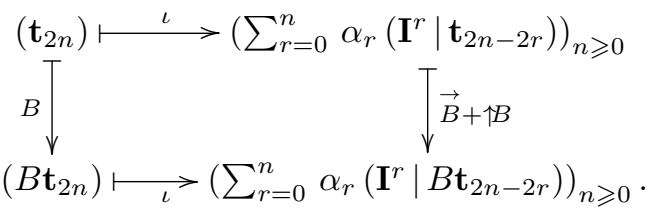

This means 6.3 also commutes with the Connes boundary maps.

We now have the characteristic map

$$
\chi \circ \cup \circ \iota: C_{\mathcal{E}(1)}^{i}\left(\Delta^{\bullet}\right) \longrightarrow C_{\mathcal{E}(1)}^{i}\left(C^{\bullet}(\mathcal{A})\right) .
$$

In fact, following the estimation given in [10, Lemma 2.1], it is not difficult to see that

$$
\chi \circ \cup \circ \iota(\varphi) \in C_{\varepsilon}^{i}\left(C^{\bullet}(\mathcal{A})\right) \subset C_{\mathcal{E}(1)}^{i}\left(C^{\bullet}(\mathcal{A})\right) .
$$

In the next step, we are going to consider the image of the cocycle (5.4) under this characteristic map. Explicitly, we are going to observe that the pairing between the image of the cocycle (5.4) and the (topological) K-theory of the algebra $\mathcal{A}$ yields the index of the Fredholm module $(\mathcal{H}, \not D)$ up to a non-zero constant. The pairing between the entire cyclic cohomology and the K-theory is established in [2, Theorem 8] which we recall below.

TheOrem 6.5. Let $\phi:=\left(\phi_{2 n}\right)_{n \geqslant 0} \in C_{\varepsilon}^{0}(\mathcal{A})$, and

$$
F_{\phi}(x):=\sum_{n \geqslant 0} \frac{(-1)^{n}(2 n) !}{n !} \phi_{2 n}(x, x, \ldots, x)
$$

be the corresponding entire function on $M_{\infty}(\mathcal{A})$. Then the additive map

$$
\langle\phi,\rangle: K_{0}(\mathcal{A}) \longrightarrow \mathbb{C}, \quad[p] \mapsto\langle\phi,[p]\rangle:=F_{\phi}(p)
$$

depends only the class $[\phi] \in H P_{\varepsilon}^{0}(\mathcal{A})$.

We now present the main result of this subsection in the discussion below. 
Let us introduce the element $\mathbf{v}=\left(v_{n}\right)_{n \in \mathbb{N}} \in C_{\mathcal{E}(1)}^{0}\left(\Delta^{\bullet}\right)$,

$$
v_{n}:=\delta_{0}^{2 n}(*)=(\underbrace{0, \ldots, 0}_{2 n \text { times }}) .
$$

We do not claim that $\mathbf{v}$ is a cocycle, but it will be useful nonetheless.

Lemma 6.6. For any idempotent $[p] \in K_{0}(\mathcal{A})$ that acts on the Hilbert space $\mathcal{H}$ as a self-adjoint operator, and $[\not D, p]=0$, we have

$$
\langle\chi \circ \cup \circ \iota(\mathbf{v}),[p]\rangle=\sum_{n \in \mathbb{N}} \frac{(-1)^{n}}{n !} \operatorname{ind}\left(\not D_{p}\right) .
$$

Proof. From the definition of the characteristic homomorphisms, and Theorem 6.5 it follows that

$$
\begin{aligned}
\langle\chi \circ \cup \circ \iota(\mathbf{v}),[p]\rangle & =\sum_{n \in \mathbb{N}} \frac{(-1)^{n}(2 n) !}{n !}\left(\sum_{r=0}^{n} \alpha_{r}\right) \operatorname{Str}(p e(0) p e(0) \cdots e(0) p e(1)) \\
& =\sum_{n \in \mathbb{N}} \frac{(-1)^{n}}{n !} \operatorname{Str}\left(p e^{-\not D^{2}}\right) .
\end{aligned}
$$

On the other hand,

$$
\operatorname{Str}\left(p e^{-\not D^{2}}\right)=\operatorname{Str}\left(p e^{-\not D^{2}} p\right)=\operatorname{Str}\left(p e^{-\not D_{p}^{2}} p\right),
$$

where $\not D_{p}=p \not D p$, and thus the claim follows from the McKean-Singer formula [10, Lemma 3.1].

We are ready to state our main result.

THEOREM 6.7. Let $(\mathcal{H}, \not D)$ be a Fredholm module over $\mathcal{A}$, and $\varphi \in C_{\mathcal{E}(1)}^{0}\left(\Delta^{\bullet}\right)$ be the $\mathcal{E}(1)$-asymptotic cyclic cocycle given by (5.4). Furthermore, let $\phi \in C_{\varepsilon}^{0}(\mathcal{A})$ be given by $\phi=\chi \circ \cup \circ \iota(\varphi)$, and $\not D_{p}:=p \not D p$. Then

$$
\langle[\phi],[p]\rangle=\left(\sum_{n \in \mathbb{N}} \frac{n+1}{2^{n}(n !)^{2}}\right) \operatorname{ind}\left(\not \phi_{p}\right) .
$$

Proof. Along the lines of [10] we may choose the idempotent $[p] \in K_{0}(\mathcal{A})$ as in Lemma 6.6. Then using (5.4, 6.5), Lemma 6.6 and Theorem 6.5, we get

$$
\begin{aligned}
\langle\phi,[p]\rangle & =\langle\chi \circ \cup \circ \iota(\varphi),[p]\rangle \\
& =\sum_{n \in \mathbb{N}} \frac{(-1)^{n}(2 n) !}{n !} \frac{(-1)^{n}}{2^{n} n !}(n+1)\left(\sum_{r=0}^{n} \alpha_{r}\right) \operatorname{ind}\left(\not D_{p}\right) \\
& =\left(\sum_{n \in \mathbb{N}} \frac{n+1}{2^{n}(n !)^{2}}\right) \operatorname{ind}\left(\not D_{p}\right) .
\end{aligned}
$$

as we wanted to show.

6.5. Odd index cocycle. Given an odd theta-summable Fredholm module $(\mathcal{H}, \not D)$ over an algebra $\mathcal{A}$, we shall construct an odd asymptotic cocycle on $\mathcal{A}$, using once again the cocycle (5.4, such that the pairing with a unitary $g \in K_{1}(\mathcal{A})$ yields the spectral flow $\operatorname{sf}\left(\not D, g^{-1} \not D g\right)$. 
Since (5.4) is an even cocycle, while we need an odd one, we have to begin with the following embedding.

Proposition 6.8. We have a morphism of differential graded $\mathbb{C}$-vector spaces of the form

$$
\eta: C_{\mathcal{E}(1)}^{*}\left(\Delta^{\bullet}\right) \longrightarrow C_{\mathcal{E}(1)}^{1}\left(C^{\bullet}(\mathcal{P})\right) \widehat{\otimes} C_{\mathcal{E}(1)}^{*}\left(\Delta^{\bullet}\right)
$$

given by

$$
\left(\mathbf{t}_{2 n}\right)_{n \geqslant 0} \mapsto\left(X \mid \mathbf{t}_{2 n}\right)_{n \geqslant 0} \quad \text { and } \quad\left(\mathbf{t}_{2 n+1}\right)_{n \geqslant 0} \mapsto\left(X \mid \mathbf{t}_{2 n+1}\right)_{n \geqslant 0}
$$

Proof. The claim follows, similar to Proposition 6.4 from $X \in H C_{\mathcal{E}(1)}^{1}(\mathcal{P} ; \epsilon, 1)$ being a cyclic 1-cocycle.

On the next move, we transform this cocycle to an odd cocycle on the algebra via a characteristic homomorphism similar to the one given by Proposition 6.3

Proposition 6.9. Let $(\mathcal{H}, \not D)$ be an odd theta-summable Fredholm module over an algebra $\mathcal{A}$, let $g \in K_{1}(\mathcal{A})$ be a unitary, and $\not D_{u}:=(1-u) \not D+u g^{-1} \not D g$. Let also $\mathcal{A}$ be a $\mathcal{P}$-module algebra for a Hopf-algebra $\mathcal{P}$ with the modular pair in involution $(\varepsilon, 1)$. Assume further that the trace $\operatorname{Tr}$ is invariant under the $\mathcal{P}$-action, and that $\mathcal{P}$ acts on $e(t):=e^{-t \not_{u}^{2}}$ trivially, for all $t \geqslant 0$. Then there is a characteristic homomorphism of cocyclic objects given by $\chi$ : $\operatorname{Diag}^{\bullet}\left(C_{\mathcal{B}}^{\bullet}(\mathcal{B} ; \epsilon, 1) \widehat{\otimes} \Delta^{\bullet}\right) \longrightarrow C^{\bullet}(\mathcal{A})$

$$
\begin{aligned}
\chi\left(h^{1} \otimes \cdots \otimes\right. & \left.h^{n} \mid t_{1}, \ldots, t_{n}\right)\left(a_{0}, \ldots, a_{n}\right) \\
& :=\operatorname{Tr}\left(a_{0} e\left(t_{1}\right) h^{1}\left(a_{1}\right) e\left(t_{2}-t_{1}\right) \ldots e\left(t_{n}-t_{n-1}\right) h^{n}\left(a_{n}\right) e\left(1-t_{n}\right)\right) .
\end{aligned}
$$

Combining with Proposition 4.1, we obtain the odd asymptotic cocycle

$$
(\chi \circ \cup \circ \eta)(\varphi) \in C_{\varepsilon}^{1}(\mathcal{A}) \subset C_{\mathcal{E}(1)}^{1}(\mathcal{A}) .
$$

We now need to pair 6.7 with the unitary $g \in K_{1}(\mathcal{A})$. To this end, we recall the odd analogue of the pairing given by Theorem 6.5 from [7]. See also [8] and [3, Section 4.7].

TheOREM 6.10. Given any $\phi:=\left(\phi_{2 n+1}\right)_{n \geqslant 0} \in C_{\varepsilon}^{1}(\mathcal{A})$, the additive map

$$
\langle\phi,\rangle: K_{1}(\mathcal{A}) \longrightarrow \mathbb{C}, \quad[g] \mapsto\langle\phi, g\rangle:=\frac{1}{\sqrt{2 \pi i}} \sum_{n=0}^{\infty}(-1)^{n} n ! \phi_{2 n+1}\left(g^{-1}, g, \ldots, g^{-1}, g\right)
$$

depends only on the class $[\phi] \in H P_{\varepsilon}^{1}(\mathcal{A})$.

We have now all the machinery we need.

THEOREM 6.11. Let $(\mathcal{H}, \not D)$ be an odd theta-summable Fredholm module over an algebra $\mathcal{A}, g \in K_{1}(\mathcal{A})$ be a unitary, and $\not D_{u}:=(1-u) \not D+u g^{-1} \not D g$. Then,

$$
\langle(\chi \circ \cup \circ \eta)(\varphi), g\rangle=\left(\frac{1}{\sqrt{2 i}} \sum_{n=1}^{\infty} \frac{n+1}{2^{n}}\right) \operatorname{Tr}\left(g^{-1} X(g) e(1)\right) .
$$

Proof. We observe that

$$
\begin{aligned}
& \langle(\chi \circ \cup \circ \eta)(\varphi), g\rangle \\
& \quad=\frac{1}{\sqrt{2 \pi i}} \operatorname{Tr}\left(g^{-1} X(g) e(1)\right)+\frac{1}{\sqrt{2 \pi i}} \sum_{n=1}^{\infty} \sum_{r=0}^{n} \frac{(-1)^{n}}{2^{n} n !}(-1)^{n} n ! \operatorname{Tr}\left(g^{-1} X(g) e(1)\right),
\end{aligned}
$$


since

$$
\begin{aligned}
\operatorname{Tr}\left(g^{-1} e(0) X_{(1)}(g) \ldots e(0) X_{(2(n-r)+1)}(g) e(1) X_{(2(n-r)+2)}\left(g^{-1}\right) e(0)\right. & \left.\ldots X_{(2 n+1)}(g) e(0)\right) \\
& =\operatorname{Tr}\left(g^{-1} X(g) e(1)\right)
\end{aligned}
$$

for any $0 \leqslant r \leqslant n$.

Finally, integrating on $u \in[0,1]$,

$$
\begin{aligned}
\int_{0}^{1}\langle\chi \circ \cup \circ \eta(\varphi), g\rangle d u & =\frac{1}{\sqrt{2 \pi i}} \sum_{n=1}^{\infty} \frac{n+1}{2^{n}} \int_{0}^{1} \operatorname{Tr}\left(\not \dot{D}_{u} e^{-\not D_{u}^{2}}\right) d u \\
& =\left(\frac{1}{\sqrt{2 i}} \sum_{n=1}^{\infty} \frac{n+1}{2^{n}}\right) \operatorname{sf}\left(\not D, g^{-1} \not D g\right)
\end{aligned}
$$

we obtain the spectral flow $\operatorname{sf}\left(\not D, g^{-1} \not D g\right)$, up to a constant multiple.

\section{References}

[1] M. F. Atiyah, V. K. Patodi, I. M. Singer, Spectral asymmetry and Riemannian geometry. III, Math. Proc. Cambridge Philos. Soc. 79 (1976), 71-99.

[2] A. Connes, Entire cyclic cohomology of Banach algebras and characters of $\theta$-summable Fredholm modules, K-Theory 1 (1988), 519-548.

[3] A. Connes, Noncommutative Geometry, Academic Press, San Diego, 1994.

[4] A. Connes, N. Higson, Déformations, morphismes asymptotiques et $K$-théorie bivariante, C. R. Acad. Sci. Paris Sér. I Math. 311 (1990), 101-106.

[5] A. Connes, H. Moscovici, Hopf algebras, cyclic cohomology and the transverse index theorem, Comm. Math. Phys. 198 (1998), 199-246.

[6] A. Connes, H. Moscovici, Cyclic cohomology and Hopf algebras, Lett. Math. Phys. 48 (1999), 97-108.

[7] J. Cuntz, D. Quillen, Algebra extensions and nonsingularity, J. Amer. Math. Soc. 8 (1995), 251-289.

[8] E. Getzler, The odd Chern character in cyclic homology and spectral flow, Topology 32 (1993), 489-507.

[9] E. Getzler, J. D. S. Jones, The cyclic homology of crossed product algebras, J. Reine Angew. Math. 445 (1993), 161-174.

[10] E. Getzler, A. Szenes, On the Chern character of a theta-summable Fredholm module, J. Funct. Anal. 84 (1989), 343-357.

[11] N. Higson, Categories of fractions and excision in KK-theory, J. Pure Appl. Algebra 65 (1990), 119-138.

[12] A. Jaffe, A. Lesniewski, K. Osterwalder, Quantum K-theory. I. The Chern character, Comm. Math. Phys. 118 (1988), 1-14.

[13] C. Kassel, Cyclic homology, comodules, and mixed complexes, J. Algebra 107 (1987), 195-216.

[14] M. Khalkhali, On the entire cyclic cohomology of Banach algebras, Thesis (Ph.D.)-Dalhousie University (Canada), ProQuest LLC, Ann Arbor, MI, 1991.

[15] M. Khalkhali, B. Rangipour, On the generalized cyclic Eilenberg-Zilber theorem, Canad. Math. Bull. 47 (2004), 38-48. 
[16] S. Klimek, A. Lesniewski, A note on the entire cyclic cohomology of a finite-dimensional noncommutative space, Canad. Math. Bull. 36 (1993), 449-457.

[17] J. Kustermans, J. Rognes, L. Tuset, The Connes-Moscovici approach to cyclic cohomology for compact quantum groups, K-Theory 26 (2002), 101-137.

[18] J. L. Loday, Cyclic Homology, second ed., Grundlehren Math. Wiss. 301, Springer, Berlin, 1998.

[19] M. Puschnigg, Asymptotic Cyclic Cohomology, Lecture Notes in Math. 1642, Springer, Berlin, 1996.

[20] D. Quillen, Higher algebraic K-theory. I, in: Algebraic K-Theory, I. Higher $K$-theories (Seattle, 1972), Lecture Notes in Math. 341, Springer, Berlin, 1973, 85-147.

[21] R. A. Ryan, Introduction to Tensor Products of Banach Spaces, Springer Monogr. Math., Springer, London, 2002.

[22] C. A. Weibel, An Introduction to Homological Algebra, Cambridge Stud. Adv. Math. 38, Cambridge Univ. Press, Canbridge, 1994. 